\title{
EVOLUTION OF THE STELLAR-TO-DARK MATTER RELATION: SEPARATING STAR-FORMING AND PASSIVE GALAXIES FROM $z=1$ TO 0
}

\author{
Jeremy L. Tinker ${ }^{1}$, Alexie Leauthaud ${ }^{2}$, Kevin Bundy $^{2}$, Matthew R. George ${ }^{3}$, Peter Behroozi ${ }^{4}$, \\ RichaRd MasseY ${ }^{5}$, Jason RHOdes ${ }^{6,7}$, AND Risa H. WeChSLER ${ }^{4}$ \\ ${ }^{1}$ Center for Cosmology and Particle Physics, Department of Physics, New York University, 4 Washington Place, \\ New York, NY 10003, USA; jeremy.tinker@ nyu.edu \\ ${ }^{2}$ Kavli Institute for the Physics and Mathematics of the Universe (Kavli IPMU, WPI), Todai Institutes \\ for Advanced Study, the University of Tokyo, Kashiwa 277-8583, Japan \\ ${ }^{3}$ Department of Astronomy, University of California, and Lawrence Berkeley National Laboratory, Berkeley, CA 94720, USA \\ ${ }^{4}$ Kavli Institute for Particle Astrophysics and Cosmology, Physics Department, Stanford University, and SLAC \\ National Accelerator Laboratory, Stanford, CA 94305, USA \\ ${ }^{5}$ Institute for Computational Cosmology, Durham University, South Road, Durham, DH1 3LE, UK \\ ${ }^{6}$ California Institute of Technology, MC 350-17, 1200 East California Boulevard, Pasadena, CA 91125, USA \\ ${ }^{7}$ Jet Propulsion Laboratory, California Institute of Technology, Pasadena, CA 91109, USA \\ Received 2013 June 3; accepted 2013 September 3; published 2013 November 8
}

\begin{abstract}
We use measurements of the stellar mass function, galaxy clustering, and galaxy-galaxy lensing within the COSMOS survey to constrain the stellar-to-halo mass relation (SHMR) of star forming and quiescent galaxies over the redshift range $z=[0.2,1.0]$. For massive galaxies, $M_{*} \gtrsim 10^{10.6} M_{\odot}$, our results indicate that star-forming galaxies grow proportionately as fast as their dark matter halos while quiescent galaxies are outpaced by dark matter growth. At lower masses, there is minimal difference in the SHMRs, implying that the majority low-mass quiescent galaxies have only recently been quenched of their star formation. Our analysis also affords a breakdown of all COSMOS galaxies into the relative numbers of central and satellite galaxies for both populations. At $z=1$, satellite galaxies dominate the red sequence below the knee in the stellar mass function. But the number of quiescent satellites exhibits minimal redshift evolution; all evolution in the red sequence is due to low-mass central galaxies being quenched of their star formation. At $M_{*} \sim 10^{10} M_{\odot}$, the fraction of central galaxies on the red sequence increases by a factor of 10 over our redshift baseline, while the fraction of quenched satellite galaxies at that mass is constant with redshift. We define a "migration rate" to the red sequence as the time derivative of the passive galaxy abundances. We find that the migration rate of central galaxies to the red sequence increases by nearly an order of magnitude from $z=1$ to $z=0$. These results imply that the efficiency of quenching star formation for centrals is increasing with cosmic time, while the mechanisms that quench the star formation of satellite galaxies in groups and clusters is losing efficiency.
\end{abstract}

Key words: cosmology: observations - galaxies: evolution - galaxies: halos

Online-only material: color figures

\section{INTRODUCTION}

One of the defining characteristics of the $z=0$ galaxy distribution is its bimodality. Galaxies can be roughly categorized into the star-forming (SF) sequence of blue, disky, gas-rich galaxies, and the quiescent, ellipsoidal galaxies with old stellar populations and red colors (Strateva et al. 2001; Blanton et al. 2003; Kauffmann et al. 2003; Madgwick et al. 2003). This bimodality is firmly in place at $z=1$ (Bell et al. 2004; Cooper et al. 2006; Willmer et al. 2006) and extends out to $z=2$ and possibly beyond (Kriek et al. 2008; Williams et al. 2009). The physical processes that drive the creation and evolution of the red sequence are not fully understood. There are many possible routes to the red sequence, but the relative efficiency of each are unquantified. In this paper we use measurements of the stellar mass function (SMF), galaxy clustering, and galaxy-galaxy lensing from the COSMOS survey (Scoville et al. (2007)) to disentangle the various process that attenuate star formation in galaxies. This paper is an extension of Leauthaud et al. (2011, 2012, hereafter L11 and L12). In L11 we presented our theoretical framework; in L12 we applied this framework to stellar mass defined samples in COSMOS; in this paper we extend this framework to samples defined by both stellar mass and star formation activity, and apply it once again to COSMOS data.
The proposed mechanisms for quenching star formation can be grouped into two broad categories: processes that affect galaxies that exist at the center of the potential well of their host dark matter halo, and processes that affect galaxies that orbit as satellites within a larger dark matter potential. Central galaxy processes include mergers, active galactic nucleus (AGN) feedback - triggered either by mergers or by disk instabilities-and shock heating of infalling gas at a critical halo mass scale or galaxy mass scale (e.g., Croton et al. 2006; Bower et al. 2006; Dekel \& Birnboim 2006; Cattaneo et al. 2006; Hopkins et al. 2008). Satellite galaxy processes do include some AGN and merging activity, but are likely dominated by tidal effects from the host dark matter halo, harassment by other galaxies within the group, strangulation from an active cold gas supply, and ram pressure stripping of gas by interaction with the host halo's hot gas (e.g., Gunn \& Gott 1972; Moore et al. 1998; Balogh et al. 2000).

In this work we define a galaxy group as a set of galaxies that share a common dark matter halo. Close pairs of halos certainly exist in the field (e.g., the Milky Way-Andromeda pair), but by our definition these are not galaxy groups. This definition matches up to the division of processes that quench galaxies defined in the previous paragraph: ram pressure, tidal stripping, and strangulation do not significantly affect galaxies until they 
have crossed the virial radius of a larger halo. ${ }^{8}$ This definition also fits seamlessly with our theoretical framework for analyzing the clustering and lensing of galaxies.

To disentangle the relative numbers of central and satellite galaxies, we use the framework of the halo occupation distribution (HOD; see, e.g., Peacock \& Smith 2000; Seljak 2000; Scoccimarro et al. 2001; Cooray \& Sheth 2002; Berlind \& Weinberg 2002 for early works, and Zheng et al. 2007; van den Bosch et al. 2007; Tinker et al. 2010b for examples of more recent implementations of the framework). In brief, the HOD provides a statistical framework for the probability distribution function of galaxies within halos. Traditionally, HOD models parameterize $P\left(N \mid M_{h}\right)$, the probability that a halo of mass $M$ contains $N$ galaxies in a pre-defined sample. The HOD for a given galaxy mass, $M_{*}$, is based on two characteristic halo mass scales: the mean halo mass for central galaxies and a larger halo mass where there is (on average) one satellite galaxy of mass $M \geqslant M_{*}$. Here we use an extended model that parameterizes this probability as a function of galaxy mass, $P\left(N \mid M_{h}, M_{*}\right)$, rather than for a specified threshold. The specific model we implement is described in detail in L11, which begins with parameterization of the stellar mass-to-halo mass relation for central galaxies (SHMR). This function specifies the mean mass of a central galaxy as a function of halo mass. The halo mass scale for satellite galaxies is motivated by previous HOD analyses that find a tight relation between these two halo mass scales.

The benefit of the COSMOS survey for this work is that it provides a consistent set of observations and the same definition of stellar mass at various redshifts. Additionally, the broad wavelength coverage of COSMOS are highly efficient at differentiating dusty SF galaxies from truly passive objects. Although data exists at multiple epochs from various surveys, the clustering of galaxies depend sensitively on survey selection (Sánchez \& Cole 2008), and stellar mass estimates depend on both survey parameters and on assumptions in the stellar mass modeling (Conroy et al. 2009; Conroy \& Gunn 2010). Constraints on the redshift evolution in the SHMR are significantly weakened when incorporating such uncertainties into the analysis (Behroozi et al. 2010), thus the COSMOS data set is crucial for identifying true redshift trends.

In this paper we will interchangeably use the terms "quiescent," "quenched," and "passive" to refer to galaxies that have little to no star formation and are intrinsically located on the red sequence. Galaxies that appear red due to dust contamination of broadband colors are included in the SF sequence. We will discuss this further in Section 2. We will refer to the "red sequence" to mean the set of galaxies that are intrinsically red. The complement of the red sequence is the set of SF galaxies. We will use the knee in the SMF, approximately $10^{10.6} M_{\odot}$ at all redshifts considered (Drory et al. 2009; Marchesini et al. 2009), as the reference point between "high-mass" and "lowmass" galaxy samples. Our reference point for small and large distance scales is $1 \mathrm{Mpc}$ (comoving; $\sim 110 \operatorname{arcsec}$ at $z=0.5$ ), which is the at the center of the transition in the galaxy correlation function from pair counts being dominated by galaxies in two distinct halos and pairs that arise from two galaxies occupying a common halo. We will frequently refer to both the fraction

\footnotetext{
8 Some studies have found an increased fraction of quenched galaxies extending several virial radii outside of clusters (e.g., Balogh et al. 2000; Hansen et al. 2009; von der Linden et al. 2010), but these results are easily explained by accounting for galaxies that are satellites in nearby groups, as well as galaxies in the cluster infall region that have orbited within the virial radius of the cluster, but the apocenter of their orbit is outside $R_{\text {vir }}$ (Wetzel et al. 2013b).
}

of galaxies that are satellites, $f_{\text {sat }}$, the fraction of galaxies that are quenched $f_{q}$, and combinations of both. For clarity, the fraction of satellites that are quenched is referenced as $f_{q}$ (sat) while the fraction of quenched galaxies that are satellites is referenced as $f_{\text {sat }}(q)$-i.e., the subsample for which the fraction is determined is referenced parenthetically, while the quantity by which the fraction is determined is listed in the subscript.

In all theoretical modeling we assume a flat $\Lambda \mathrm{CDM}$ cosmological model of $\left(\Omega_{m}, \sigma_{8}, \Omega_{b}, n_{s}, h_{0}\right)=(0.272,0.807,0.0438$, $0.963,0.72)$. We define a dark matter halo as a spherical, virialized object with a mean interior density of $\Delta \equiv$ $3 M_{h} / 4 \pi \Omega_{m} \rho_{\text {crit }} R_{h}^{3}=200$. All halo statistics used in this paper are calibrated from numerical simulations that match this halo definition.

\section{DATA}

Details of the COSMOS survey can be found in Scoville et al. (2007). Details of the measurement techniques and methods for the SMFs, angular galaxy clustering $\left(w_{\theta}\right)$, and galaxy-galaxy lensing $(\Delta \Sigma)$ can be found in L12. All $w_{\theta}$ measurements are taken from the Subaru catalog $\left(2.3 \mathrm{deg}^{2}\right)$ while lensing and SMF measurements are restricted to the HST ACS catalog $\left(1.64 \mathrm{deg}^{2}\right)$. The sample selection is also identical to L12. Here we repeat all these measurements, now broken into two subsamples of SF (blue) and passive (red) objects. Intrinsically passive galaxies are identified in a specific region in the $(\mathrm{NUV}-R)-(R-J)$ color-color space in the same manner as Bundy et al. (2010). The addition of near-IR data breaks the degeneracy between dusty and SF objects (Pozzetti \& Mannucci 2000; Labbé et al. 2005; Williams et al. 2009; Zhu et al. 2011).

Photometric redshifts are obtained from Ilbert et al. (2009), versions $v 1.7$ and $v 1.8$. These photo- $z$ estimates have negligible differences at $z<1$ but v1.8 has improved accuracy relative to spectroscopic redshifts. The v1.7 photo- $z$ 's are used for the SMF and $w_{\theta}$ and v1.8 for the lensing catalog. Later version of the photometric redshifts, which were not available during much of the present work, focus on $z \sim 2$. We have confirmed that there are negligible changes to $z<1$ results.

Stellar masses are estimated using the Bayesian code of Bundy et al. (2006) and assume a Chabrier (2003) initial mass function. While the method of Bundy et al. uses multi-band colors to constrain the mass-to-light $(\mathrm{M} / \mathrm{L})$ ratio in the observed $K$-band, with mass estimates derived from application to the $K$-band luminosity only.

We restrict our analysis to stellar masses above the $80 \%$ stellar mass completeness limit, as in L12. Due to the lower intrinsic luminosities at fixed stellar mass, passive galaxies have a higher completeness limit at fixed redshift by roughly $\sim 0.2 \mathrm{dex}$. For the $\mathrm{SMF}$ and the galaxy-galaxy lensing measurements, we restrict the measurements of the passive population to be above that limit (although we will compare our lensing fits to the data for these bins in the presentation of our results for comparison purposes). For the clustering measurements, we find that including passive galaxies down to the SF stellar mass limit does not bias the clustering of those samples ${ }^{9}$ thus we incorporate these galaxies in the clustering bins. See Figure 1 in L12 for a plot of the completeness limits as a function of redshift. Stellar mass limits are also given in Table 1.

\footnotetext{
9 The passive galaxy completeness limit essentially cuts part-way through the lowest stellar mass bin at each redshift interval. We compares measurements of the clustering of all passive galaxies in this bin to those that are above the limit, finding that the results are consistent with one another, but the higher number of galaxies in the full bin yields better error bars, especially at small scales.
} 
Table 1

Binning Scheme for Galaxies in $\log _{10}\left(M_{*}\right)$

\begin{tabular}{cccccccc}
\hline \hline$\Delta \Sigma$ & bin1 & bin2 & bin3 & bin4 & bin5 & bin6 & bin7 \\
\hline$z_{1}, \min$ & 11.12 & 10.89 & 10.64 & 10.3 & 9.82 & 9.2 & 8.7 \\
$z_{1}, \max$ & 12.0 & 11.12 & 10.89 & 10.64 & 10.3 & 9.8 & 9.2 \\
\hline$z_{2}, \min$ & 11.29 & 11.05 & 10.88 & 10.65 & 10.3 & 9.8 & 9.3 \\
$z_{2}, \max$ & 12.0 & 11.29 & 11.05 & 10.88 & 10.65 & 10.3 & 9.8 \\
\hline$z_{3}, \min$ & 11.35 & 11.16 & 10.97 & 10.74 & 10.39 & 9.8 & $\ldots$ \\
$z_{3}, \max$ & 12.0 & 11.35 & 11.16 & 10.97 & 10.74 & 10.39 & $\ldots$ \\
\hline$w_{\theta}$ & $\operatorname{bin} 1$ & $\operatorname{bin} 2$ & $\operatorname{bin} 3$ & $\operatorname{bin} 4$ & $\operatorname{bin} 5$ & bin6 & \\
\hline$z_{1}, \min$ & 8.8 & 9.3 & 9.8 & 10.3 & 10.8 & $\ldots$ & \\
$z_{1}, \max$ & 9.3 & 9.8 & 10.3 & 10.8 & 11.3 & $\ldots$ & \\
\hline$z_{2}, \min$ & $\ldots$ & 9.3 & 9.8 & 10.3 & 10.8 & 11.1 & \\
$z_{2}, \max$ & $\ldots$ & 9.8 & 10.3 & 10.8 & 11.3 & 11.6 & \\
\hline$z_{3}, \min$ & $\ldots$ & $\ldots$ & 9.8 & 10.3 & 10.8 & 11.1 & \\
$z_{3}, \max$ & $\ldots$ & $\ldots$ & 10.3 & 10.8 & 11.3 & 11.6 & \\
\hline
\end{tabular}

In addition to the SMFs, we also incorporate the ratio of the passive and SF SMFs into our analysis. The ratio takes into account that the amplitudes of the passive and SF SMFs are correlated to some degree, given that they are measured from the same sample of galaxies. All measurements are made in three redshift bins that span a range of $z=[0.22,1.00]$. The median redshifts are $z=0.36,0.66$, and 0.88 . We will present our measurements in Section 4 when discussing our best-fit models.

We also incorporate information from the COSMOS X-ray group catalog of George et al. (2011). The central galaxy in each group is determined with high probability, yielding a measurement of the red fraction of central galaxies at $M_{h} \sim$ $10^{13.5} M_{\odot}$ in each redshift bin. The error in this quantity is determined by bootstrap resampling of the group catalog. The purpose of including these data is to prevent unphysical divergent behavior of the models, e.g., models in which the red fraction of central galaxies turns over and approaches zero at high halo masses where the constraints from the three galaxy measures are weak. In practice, the inclusion of the group data does not significantly affect the results.

As described in L11 and L12, we use a large-volume, highresolution $\mathrm{N}$-body simulation to create mock galaxy distributions with the same angular size and comoving depth of each slice in the COSMOS survey. We use the "Consuelo" simulation, which is part of the LasDamas simulation suite (C. McBride, in preparation). This simulation is $420 \mathrm{~h}^{-1} \mathrm{Mpc}$ on a side and contains $1400^{3}$ particles. These mocks are then used to estimate the covariance matrices of each data set. Within the simulation, we are able to create 409,179 , and 105 mocks for the $z=0.36$, 0.66 , and 0.88 redshift bins, respectively. We populate the halos in the simulation with galaxies using a preliminary HOD fit to the measurements, yielding a preliminary estimate of the covariances. We then repeat this procedure with HOD fits that utilize the first covariance matrices to produce the final errors used in the results presented here. These covariance matrices are used as the full errors on the SMFs and $w_{\theta}$ measurements. Because these two statistics involve simple counting of galaxies and their pairs, the $N$-body simulations encompass both the sample variance from large-scale structure and shot noise from small number statistics. For the lensing measurements, the statistical errors arising from the ellipticity measurements of the background sources are added to the covariance matrices from the mocks, which estimate the sample variance. In most cases, the statistical errors dominate the uncertainty in $\Delta \Sigma$.

\section{THEORY}

In L11, we outlined an HOD-based model that can be used to analytically predict the SMF, galaxy-galaxy lensing, and clustering signals. A key component of this model is the SHMR which is modeled as a mean-log relation, noted as $M_{*}=$ $f_{\text {SHMR }}\left(M_{h}\right)$, with a $\log$-normal scatter ${ }^{10}$ noted $\sigma_{\log M_{*}}$. Here we give a brief review of the model of the minor modifications used to adapt it to passive and SF subsamples of galaxies.

\subsection{The Stellar-to-halo Mass Relation for Central Galaxies}

Following Behroozi et al. (2010), $f_{\text {SHMR }}\left(M_{h}\right)$ is mathematically defined following its inverse function:

$$
\begin{aligned}
\log _{10}\left(f_{\mathrm{SHMR}}^{-1}\left(M_{*}\right)\right)= & \log _{10}\left(M_{1}\right)+\beta \log _{10}\left(\frac{M_{*}}{M_{*, 0}}\right) \\
& +\frac{\left(\frac{M_{*}}{M_{*, 0}}\right)^{\delta}}{1+\left(\frac{M_{*}}{M_{*, 0}}\right)^{-\gamma}}-\frac{1}{2}
\end{aligned}
$$

where $M_{1}$ is a characteristic halo mass, $M_{*, 0}$ is a characteristic stellar mass, $\beta$ is the low-mass slope, and $\delta$ and $\gamma$ control the massive end slope. We note that Equation (1) is only relevant for central galaxies. We use Equation (1) to parameterize the SHMR of both passive and SF central galaxies, but each subsample will have a separate $f_{\text {SHMR }}$.

Equation (1) specifies the mean halo mass as a function of $M_{*}$. We assume that the distribution of central galaxy mass at fixed halo mass, $\Phi_{c}\left(M_{*} \mid M_{h}\right)$, follows a log-normal distribution with scatter $\sigma_{\log M_{*}}$. We will discuss halo occupation of central galaxies at fixed halo mass presently. Previous work suggests that $\sigma_{\log M_{*}}$ is independent of halo mass. More et al. (2011) finds a scatter in $M_{*}$ at fixed halo mass of $0.17 \pm 0.04$ dex. Moster et al. (2010) are able to fit the Sloan Digital Sky Survey (SDSS) galaxy clustering measurements assuming constant $\sigma_{\log M_{*}}$. In L12 we found that a halo mass-varying scatter produced no better fit than a model with constant scatter. We adopt a constant $\sigma_{\log M_{*}}$ here as well, but allow the scatter for passive and SF central galaxies to be independent.

\subsection{Accounting for Passive and Star-forming Subsamples}

We are bound by the requirement that each halo contains one and only one central galaxy. The mass of that galaxy may be too small to be counted in any COSMOS sample, but formally we require that

$$
\begin{aligned}
& \int f_{q}\left(M_{h}\right) \times \Phi_{\mathrm{cen}}^{\mathrm{q}}\left(M_{*} \mid M_{h}\right)+\left[1-f_{q}\left(M_{h}\right)\right] \\
& \times \Phi_{\mathrm{cen}}^{\mathrm{SF}}\left(M_{*} \mid M_{h}\right) d M_{*}=1,
\end{aligned}
$$

where $f_{q}\left(M_{h}\right)$ is a function specifying the fraction of times that a halo of mass $M_{h}$ contains a quenched central galaxy (independent of galaxy mass), and $\Phi_{\text {cen }}^{\mathrm{x}}\left(M_{*} \mid M_{h}\right)$ is the conditional SMF for central quenched or SF galaxies, each normalized to unity. Parameterizing the quenching of central galaxies by halo mass as opposed to stellar mass (or the ratio between the two) makes

\footnotetext{
10 Scatter is quoted as the standard deviation of the logarithm base 10 of the
} stellar mass at fixed halo mass. 
an implicit choice of the mechanisms that quench star formation in central galaxies (see the discussions in Hopkins et al. 2008 and Tinker \& Wetzel 2010). Given the small scatter between stellar mass and halo mass, this choice is not likely to bias the results we focus on here, e.g., the fraction of centrals that are red. This choice is also beneficial for its ease of implementation in our halo occupation framework.

We do not choose a parametric form for $f_{q}\left(M_{h}\right)$. Rather, we choose five halo mass points at which to specify $f_{q}\left(M_{h}\right)$ and smoothly interpolate between them. The five masses are evenly spaced in $\log M_{h}$ from 10.8 to 14.0 .

\subsection{Calculating Halo Occupation of Centrals and Satellites}

In order to avoid explicit dependence of our HOD parameters on our bin size, we define all HODs as threshold quantities. Having halo occupation parameterized for threshold samples yields maximal flexibility for taking the same HOD parameters and calculating $\langle N\rangle_{M}$ for a bin of arbitrary size. For a sample of galaxies above a threshold stellar mass, the central occupation function $\left\langle N_{\text {cen }}\right\rangle_{M}$ is expressed as

$$
\begin{aligned}
& \left\langle N_{\text {cen }}\left(M_{h} \mid>M_{*}\right)\right\rangle \\
& \quad=\frac{1}{2}\left[1-\operatorname{erf}\left(\frac{\log _{10}\left(M_{*}\right)-\log _{10}\left(f_{\text {SHMR }}\left(M_{h}\right)\right)}{\sqrt{2} \sigma_{\log M_{*}}}\right)\right] .
\end{aligned}
$$

As discussed in L11, Equation (3) correctly captures the behavior of $\left\langle N_{\text {cen }}\right\rangle_{M}$ for massive galaxy samples, as opposed to the common parameterization where scatter is parameterized at fixed stellar mass as opposed to fixed halo mass. Equation (3) is valid for both SF and passive central galaxies, but the parameters of the $f_{\text {SHMR }}$ are independent for each subsample. Equation (3) assumes that there is one central galaxy per halo; in the case of our subsamples, this is not explicitly true. For red central galaxies, Equation (3) is multiplied by $f_{q}\left(M_{h}\right)$, and by $1-f_{q}\left(M_{h}\right)$ for SF central galaxies.

The occupation of satellite galaxies as a function of halo mass, $\left\langle N_{\text {sat }}\right\rangle_{M}$, is

$$
\left\langle N_{\text {sat }}\left(M_{h} \mid>M_{*}\right)\right\rangle=\left(\frac{M_{h}}{M_{\text {sat }}}\right)^{\alpha_{\text {sat }}} \exp \left(\frac{-\left(M_{\text {cut }}+f_{\text {SHMR }}^{-1}\left(M_{*}\right)\right.}{M_{h}}\right),
$$

where $M_{\text {sat }}$ is the halo mass scale for satellite galaxies, $M_{\text {cut }}$ is a cutoff scale, and $\alpha_{\text {sat }}$ is how the number of satellites scales with halo mass. We treat the satellite occupation of the passive and SF subsamples independently; unlike central galaxies, there is no integral constraint on the total number of satellite galaxies any halo can have. Equation (4) is a minor modification from L11 (Equation (12) therein); in L11, $\left\langle N_{\text {sat }}\right\rangle_{M}$ is proportional to $\left\langle N_{\text {cen }}\right\rangle_{M}$ - this guarantees that satellite occupation fully cuts off at the same halo mass scale as central galaxies of the same mass. However, in our new red/blue parameterization this would correlate $\left\langle N_{\text {cen }}\right\rangle_{M}$ to $f_{q}\left(M_{h}\right)$. We circumvent this problem by including $f_{\mathrm{SHMR}}^{-1}$ to the numerator in the exponential cutoff, producing a similar cutoff scale.

HOD modeling of luminosity-dependent galaxy clustering has shown that $M_{\text {sat }}$ is roughly 20 times $f_{\text {SHMR }}^{-1}$, varying weakly with luminosity (e.g., Zehavi et al. 2005, 2011; Zheng et al. 2007, 2009). We thus parameterize $M_{\text {sat }}$ and $M_{\text {cut }}$ as

$$
\frac{M_{\mathrm{sat}}}{10^{12} M_{\odot}}=B_{\mathrm{sat}}\left(\frac{f_{\mathrm{SHMR}}^{-1}}{10^{12} M_{\odot}}\right)^{\beta_{\mathrm{sat}}}
$$

and

$$
\frac{M_{\mathrm{cut}}}{10^{12} M_{\odot}}=B_{\text {cut }}\left(\frac{f_{\mathrm{SHMR}}^{-1}}{10^{12} M_{\odot}}\right)^{\beta_{\mathrm{cut}}}
$$

In L12 we set $\alpha_{\text {sat }}=1$, in agreement with many previous results. However, the fraction of satellites that are SF depends on halo mass (Wetzel et al. 2012), thus we allow $\alpha_{\text {sat }}$ to be free for both passive and SF subsamples. Equations (3) and (4) give the number of galaxies above a mass threshold as a function of halo mass. Our data are measured in stellar mass bins. To determine the halo occupation in a given bin, we simply take the difference between $\left\langle N_{\text {sat }}\right\rangle_{M}$ (or $\left.\left\langle N_{\text {cen }}\right\rangle_{M}\right)$ at the low- and high-mass edges of the bin.

The model has 27 free parameters. To model the halo occupation of a given subsample requires 11 free parameters. The SHMR has five free parameters $\left(M_{1}, M_{*, 0}, \beta, \delta, \gamma\right)$, with one additional parameter for the scatter, $\sigma_{\log M_{*}}$. The satellite occupation requires five more parameters $\left(B_{\text {sat }}, \beta_{\text {sat }}, B_{\text {cut }}, \beta_{\text {cut }}\right.$, $\left.\alpha_{\text {sat }}\right)$. To determine the fraction of central galaxies that are red at each halo mass requires 5 more parameters for a total of 27 . Each set of 27 parameters describes the galaxy-halo relation at a given redshift. For each of our three redshift bins, we fit the parameters separately. We use the halo mass function of Tinker et al. (2008a), the halo bias relation of Tinker et al. (2010a), and the concentration-mass relation for dark matter halos of MuñozCuartas et al. (2011), assuming that satellite galaxies follow the dark matter within a halo with a Navarro-Frenk-White profile (Navarro et al. 1997). We refer the reader to L11 for a complete description of how to take the halo occupation parameters and calculate the SMFs, clustering, and lensing signals.

\section{RESULTS}

We use Markov Chain Monte Carlo (MCMC) analysis to find both the best-fit model and the uncertainties in model parameters. We analyze each redshift bin separately. For each trial model in the MCMC chain, we calculate a separate $\chi^{2}$ for the SMF, for each mass bin in $w_{\theta}$, and each mass bin in $\Delta \Sigma$, for passive and SF subsamples, and the red fraction of central galaxies within the $\mathrm{X}$-ray groups. The total $\chi^{2}$ is then

$$
\chi_{\mathrm{tot}}^{2}=\sum_{q, \mathrm{SF}}\left[\chi_{\mathrm{smf}}^{2}+\sum_{i=1}^{N_{w}} \chi_{w, i}^{2}+\sum_{j=1}^{N_{\Delta \Sigma}} \chi_{\Delta \Sigma, j}^{2}\right]+\chi_{\text {fred }}^{2}+\chi_{\text {ratio }}^{2} .
$$

The last two terms in the above equation represent the $\chi^{2}$ for the red central fraction from the X-ray group catalog and the $\chi^{2}$ for the ratio of the passive $(q)$ and SF SMFs, respectively. We use a covariance matrix for each individual $\chi^{2}$ calculation, with the exception of $\chi_{\text {fred }}^{2}$. Parameter values and errors from the MCMC chains are in Table 2. The total $\chi^{2}$ for each best-fit model is listed in Table 3.

\subsection{Stellar Mass Functions and the Quenched Fraction of Galaxies}

Figure 1 shows our measurements of the passive and SF SMFs in COSMOS. Data are shown down to the stellar mass completeness limits for each subtype. The SMFs show limited evolution across our redshift range with the exception of lowmass passive galaxies: the abundance of these galaxies increases by a factor of two to three depending on stellar mass. This trend has been shown in a number of papers as a component of the "downsizing" of galaxy formation. Brinchmann \& Ellis (2000) 

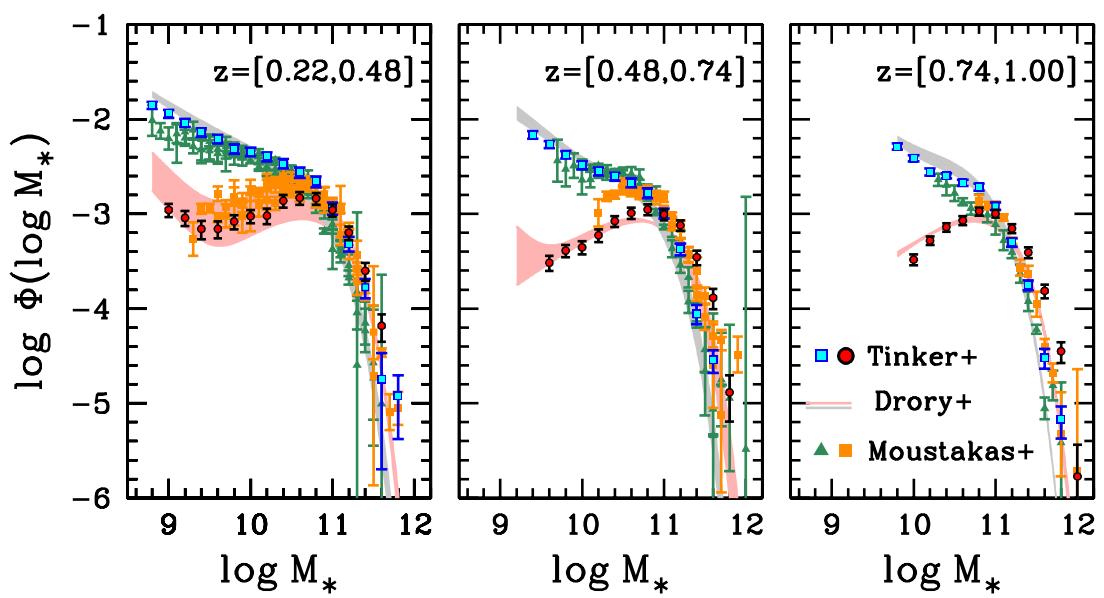

Figure 1. COSMOS stellar mass function, measured in our redshift bins, compared to other stellar mass functions at comparable redshifts. The blue squares and red circles represent our COSMOS measurements for SF and passive galaxies, respectively. The gray and pink shaded bands show the COSMOS measurements from Drory et al. (2009). Because Drory measured the SMF in different redshift bins, the bands show the range of SMF values for the two bins that overlap with each redshift bin used here. The orange squares and green triangles represent the measurements from PRIMUS (Moustakas et al. 2013) for passive and SF galaxies. In each panel, PRIMUS results are shown for all redshift bins whose median redshift is contained within the given COSMOS redshift bin (shown at the top of each panel). PRIMUS results are based on spectroscopy, thus do not go as faint as the two COSMOS results.

(A color version of this figure is available in the online journal.)

Table 2

HOD Values from MCMC

\begin{tabular}{|c|c|c|c|}
\hline Parameter & $z_{1}$ & $z_{2}$ & $z_{3}$ \\
\hline \multicolumn{4}{|c|}{ Active galaxies } \\
\hline $\log M_{1}$ & $12.56 \pm 0.05$ & $12.77 \pm 0.05$ & $12.69 \pm 0.04$ \\
\hline $\log M_{*, 0}$ & $10.96 \pm 0.06$ & $10.98 \pm 0.03$ & $10.97 \pm 0.02$ \\
\hline$\beta$ & $0.44 \pm 0.02$ & $0.46 \pm 0.03$ & $0.43 \pm 0.02$ \\
\hline$\delta$ & $0.52 \pm 0.29$ & $1.15 \pm 0.31$ & $0.73 \pm 0.25$ \\
\hline$\gamma$ & $1.48 \pm 0.43$ & $2.15 \pm 0.51$ & $4.71 \pm 0.56$ \\
\hline$\sigma_{\log M_{*}}$ & $0.21 \pm 0.06$ & $0.24 \pm 0.02$ & $0.25 \pm 0.01$ \\
\hline$B_{\text {cut }}$ & $0.28 \pm 1.91$ & $0.22 \pm 1.09$ & $0.18 \pm 1.10$ \\
\hline$B_{\text {sat }}$ & $33.96 \pm 19.61$ & $24.55 \pm 21.29$ & $112.70 \pm 26.81$ \\
\hline$\beta_{\text {cut }}$ & $0.77 \pm 1.80$ & $0.62 \pm 0.96$ & $1.00 \pm 1.42$ \\
\hline$\beta_{\text {sat }}$ & $1.05 \pm 0.44$ & $1.16 \pm 0.52$ & $2.65 \pm 0.39$ \\
\hline$\alpha_{\text {sat }}$ & $0.99 \pm 0.20$ & $0.96 \pm 0.18$ & $0.84 \pm 0.14$ \\
\hline \multicolumn{4}{|c|}{ Passive galaxies } \\
\hline $\log M_{1}$ & $12.08 \pm 0.20$ & $12.18 \pm 0.23$ & $12.21 \pm 0.17$ \\
\hline $\log M_{*, 0}$ & $10.70 \pm 0.10$ & $10.78 \pm 0.13$ & $10.83 \pm 0.10$ \\
\hline$\beta$ & $0.32 \pm 0.09$ & $0.13 \pm 0.07$ & $0.02 \pm 0.04$ \\
\hline$\delta$ & $0.93 \pm 0.25$ & $0.81 \pm 0.18$ & $0.44 \pm 0.11$ \\
\hline$\gamma$ & $0.81 \pm 0.58$ & $0.09 \pm 0.41$ & $0.81 \pm 0.23$ \\
\hline$\sigma_{\log M_{*}}$ & $0.28 \pm 0.03$ & $0.21 \pm 0.03$ & $0.18 \pm 0.05$ \\
\hline$B_{\text {cut }}$ & $21.42 \pm 10.34$ & $0.01 \pm 0.01$ & $0.21 \pm 1.42$ \\
\hline$B_{\text {sat }}$ & $17.90 \pm 22.99$ & $21.35 \pm 9.50$ & $13.16 \pm 3.83$ \\
\hline$\beta_{\text {cut }}$ & $-0.12 \pm 0.46$ & $-1.55 \pm 1.53$ & $0.46 \pm 0.80$ \\
\hline$\beta_{\text {sat }}$ & $0.62 \pm 0.52$ & $0.58 \pm 0.14$ & $0.77 \pm 0.22$ \\
\hline$\alpha_{\mathrm{sat}}$ & $1.08 \pm 0.26$ & $1.15 \pm 0.10$ & $0.98 \pm 0.12$ \\
\hline \multicolumn{4}{|c|}{ Passive central fraction } \\
\hline $\log f_{q}\left(M_{1}\right)$ & $-1.28 \pm 0.20$ & $-7.32 \pm 2.32$ & $-6.89 \pm 2.18$ \\
\hline $\log f_{q}\left(M_{2}\right)$ & $-0.85 \pm 0.10$ & $-1.17 \pm 1.04$ & $-1.23 \pm 1.47$ \\
\hline$f_{q}\left(M_{3}\right)$ & $0.54 \pm 0.07$ & $0.47 \pm 0.10$ & $0.43 \pm 0.09$ \\
\hline$f_{q}\left(M_{4}\right)$ & $0.63 \pm 0.05$ & $0.68 \pm 0.07$ & $0.59 \pm 0.08$ \\
\hline$f_{q}\left(M_{5}\right)$ & $0.77 \pm 1.36$ & $0.81 \pm 0.20$ & $0.76 \pm 0.17$ \\
\hline
\end{tabular}

detected this trend in morphologically selected samples, and Bundy et al. (2006) found similar results in the abundances of $\mathrm{SF}$ and passive galaxies in DEEP2. In our measurements, the $z=0.36$ passive SMF shows a minimum at $M_{*} \sim 10^{9.5} M_{\odot}$, with a subsequent upturn at lower masses, as shown by Drory
Table 3

$\chi^{2}$ Values for Best-fit Models

\begin{tabular}{lcc}
\hline \hline$z=[0.22,0.48]$ & $z=[0.48,0.74]$ & $z=[0.74,1.00]$ \\
\hline $218.5 /(247-27)$ & $273.0 /(241-27)$ & $220.5 /(207-27)$ \\
\hline
\end{tabular}

Note. The value in each column is the $\chi^{2}$ value divided by the number of data points minus the number of free parameters.

et al. (2009) for COSMOS data and confirmed in PRIMUS by Moustakas et al. (2013).

In Figure 1 we compare our measurements to those from Drory et al. (2009) and Moustakas et al. (2013). The Drory et al. (2009) measurements are also taken from COSMOS, but with two main differences. First, they are measured in different redshift bins. Due to the small footprint of COSMOS, the sample variance from different binning is a non-negligible effect. Second, there are differences in the stellar mass calculations themselves: Drory et al. fit the $\mathrm{M} / \mathrm{L}$ ratio from all photometric bands, while the method of Bundy et al. uses multi-band colors to constrain the $\mathrm{M} / \mathrm{L}$ ratio in the observed $K$-band, with mass estimates derived from application to the $K$-band luminosity only. There are also minor differences in the stellar population templates used. Last, in this figure we plot the fitting function results rather than the measurements themselves. The Drory et al. measurements lie slightly above their fits at the massive end, so the agreement with our data is somewhat better than implied in this figure. Even so, there are minimal differences in the SMFs.

The SMFs from Moustakas et al. (2013) are measured from PRIMUS (their Figure 11; tabulated data kindly provided by J. Moustakas), which is a larger area but does not go as deep as COSMOS due to the use of low-resolution spectroscopy to obtain galaxy redshifts. The abundance of passive galaxies is somewhat higher in the PRIMUS results, but the conclusion of Moustakas et al. (2013) agrees with our measurements here: that the only significant change in abundance is in the low-mass passive population.

Because the focus of this paper is on the growth of the red sequence, we compare our measurements for the redshift 


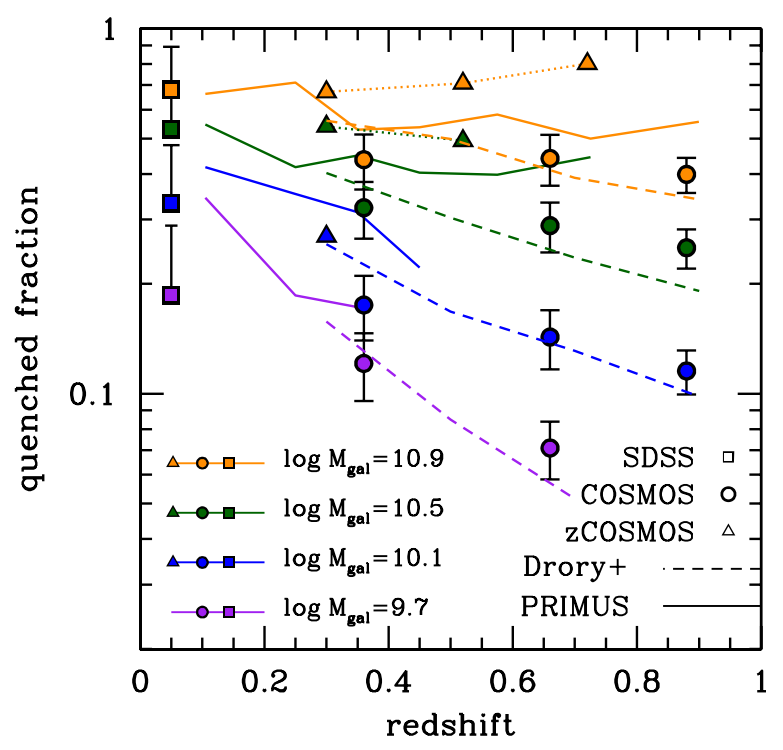

Figure 2. Comparison of the evolving quenched fractions in COSMOS (from this paper), COSMOS (from Drory et al. 2009), PRIMUS (Moustakas et al. 2013), and zCOSMOS (Knobel et al. 2013) and SDSS. Circles with errors are the COSMOS data in this paper from Figure 3, which use a NUV $-R-J$ color diagram to isolate passive galaxies. Squares are from a volume-limited SDSS sample that uses $D_{n} 4000$ to determine $f_{q}$. This sample will be used later in the paper and is discussed in Section 4.3. The dashed lines show the results from the Drory et al. (2009) COSMOS measurements, which are from the same raw data but use different methods to determine stellar mass. The PRIMUS results use a different estimate of stellar mass and use SED fitting to determine the delineation between passive and active galaxies. The zCOSMOS results use a single $U-B$ color cut to determine the set of passive galaxies.

(A color version of this figure is available in the online journal.)

evolution of the overall quenched fraction to that of recent measurements from PRIMUS (Moustakas et al. 2013) and to the analysis of zCOSMOS by Knobel et al. (2013). We define the quenched fraction as the density of passive galaxies relative to the total number. PRIMUS contains within it the COSMOS field, but there are differences in both the stellar mass assignment and in the determination of which galaxies are passive. Moustakas et al. (2013) use spectral energy distribution (SED) fitting to estimate the star formation rates of PRIMUS objects and then divide the sample based upon this distribution. Figure 2 compares the quenched fractions for four different stellar masses between the two surveys. At all masses, the PRIMUS $f_{q}$ is slightly higher than the COSMOS value(s). An important comparison, however, is the slope of $f_{q}$ with redshift. For each bin in $M_{*}$, the rate of change appears consistent between the two surveys. For zCOSMOS, the flux limit makes it difficult to achieve a long redshift baseline for anything but the most massive galaxies. But the quenched fractions in Knobel et al. (2013) are significantly higher than either PRIMUS or this work. Knobel et al. (2013) use a $U-B$ color cut to select their sample of passive galaxies, which may be susceptible to dust contamination. In their paper they compare their quenched fractions to those derived from a NUV $-R-J$ color-color diagram (similar to the approach used here), finding very good agreement. In contrast to their results (their Figure 1), the singlecolor cut is not consistent with the NUV $-R-J$ color selection and it yields a decreasing $f_{q}$ with decreasing $z$ at the most massive galaxies, which is at odds with the other two results. We will make further comparisons with the results of Knobel et al. (2013) in Section 4.5.

In this figure we have included data from the SDSS groups catalog of Tinker et al. (2011). These represent the data points at $z=0.05$. In this figure, we are presenting $f_{\mathrm{Q}}$ for the overall galaxy population, but the group finder is applied to volumelimited samples derived from the SDSS Main sample, yielding a full central-satellite decomposition of all galaxies in the sample. This group catalog is $\sim 95 \%$ complete in finding central galaxies and $\sim 90 \%$ pure in its sample of satellite galaxies. Quenched fractions in sub-populations of the group catalog are corrected for impurity and completeness statistically (see further details in Tinker et al. 2011). We will make significant use of this catalog later in the paper. The differences in stellar mass estimates between COSMOS and SDSS make comparisons of absolute abundances problematic, but fractions are more robust. To facilitate a more robust comparison of the SDSS data with our COSMOS results, we have added 0.2 dex to the stellar mass estimates and added 0.2 dex of scatter. The former represents the 0.2 dex shift in the SMFs between SDSS (Li \& White 2009) and COSMOS once deconvolved to a common scatter value. The latter is meant to mock up the increase uncertainties between SDSS spectroscopic redshifts and COSMOS photometric redshifts (see the discussion in Figure 14 in L12). Both of these changes combine to reduce $f_{\mathrm{Q}}$ by 0.1 to 0.2 , with the shift in mass scale dominating the effect. The upper error bars show the original SDSS values before shifting and adding scatter. Because both alterations to the SDSS data lower $f_{\mathrm{Q}}$, the values used here should be considered lower limits on the quenched fraction of SDSS galaxies. We note that the comparison with both PRIMUS and $\mathrm{zCOSMOS} f_{\mathrm{Q}}$ values may also be affected by differences in the stellar mass estimates as opposed to definitions of quenched. zCOSMOS, and to a lesser extent PRIMUS, has higher redshift accuracy than COSMOS, thus the scatter in $M_{*}$ will be smaller. Offsets in the stellar masses can come into play here as well.

\subsection{Comparison of the Measurements to the Best Model Fits}

Figure 3 compares the SMFs to the best-fit halo occupation models from the MCMC chains. The overall model SMF is shown with the thick solid curves, and the contribution to the SMFs from satellite galaxies is shown with the thin curves. The lower panels show the abundance ratio of SF and passive galaxies. In these panels, the growth of the red sequence at low mass is more evident.

Figure 4 shows the clustering measurements for the passive and SF galaxies. Consistent with previous measurements from other redshifts and other surveys, the passive galaxies have equal or higher clustering than the SF galaxies at every bin of stellar mass. For low-mass galaxies, the enhanced clustering of passive galaxies is due to the high fraction of such galaxies being satellites in high-mass halos (e.g., Zehavi et al. 2005; Tinker et al. 2008b; van den Bosch et al. 2003; Skibba \& Sheth 2009; Weinmann et al. 2006; Tinker \& Wetzel 2010; Wetzel et al. 2012). This effect gives rise to the well-known color-density relation. At high masses, $\log M_{*} \geqslant 10.8$, the large-scale bias appears roughly independent of color, while the small-scale clustering of passive galaxies is slightly enhanced. As we will see when inspecting the constraints on the SHMR in Figures 7, massive SF galaxies live in higher mass halos than their red counterparts, when binned by halo mass; this is true of both the SHMR results presented here and the group catalog results from Tinker et al. (2012, hereafter T12). However, when binned by galaxy mass, scatter minimizes the difference in the mean halo mass and thus the large-scale bias. Massive SF galaxies have nearly negligible satellite fractions in comparison to massive passive galaxies (at least at $z \geqslant 0.48$ ), yielding 

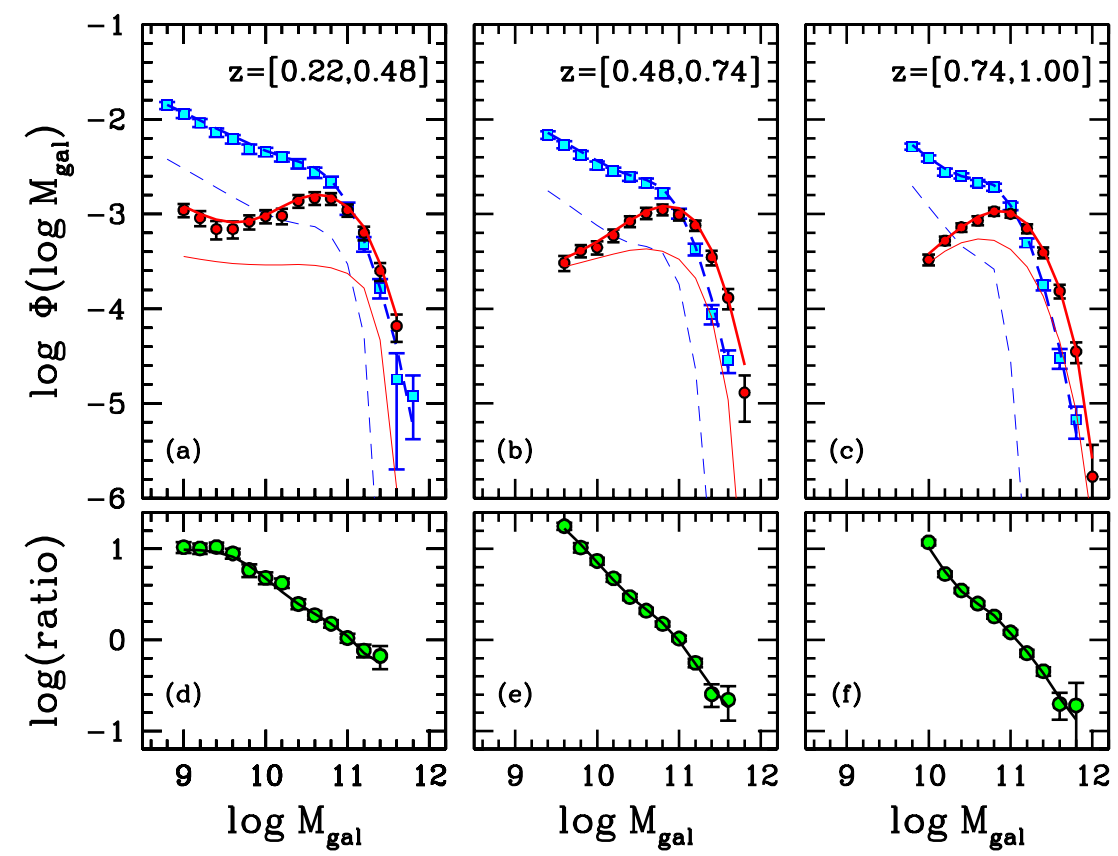

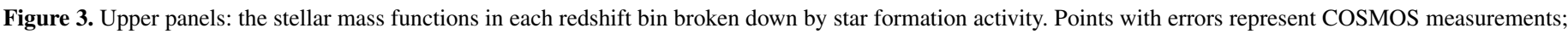

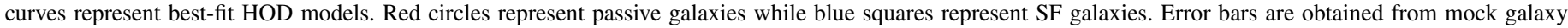

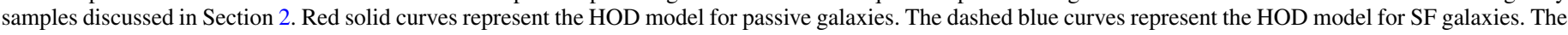

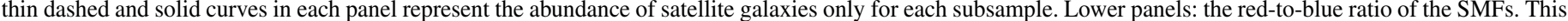

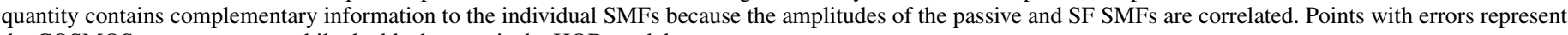
the COSMOS measurements, while the black curve is the HOD model.

(A color version of this figure is available in the online journal.)

a higher amplitude for the passive galaxy subsample at small scales.

Figure 5 rounds out our presentation of the data and model fits. Lower-mass SF galaxies primarily live as central galaxies in lower mass halos (see also Velander et al. 2013), the lensing signal is weaker than that of passive galaxies and thus has larger statistical errors. This is reflected in the large error bars for the lower-mass SF measurements. To better understand the information that the lensing signal affords, Figure 6 shows a breakdown of the constituent parts of the lensing fit for high mass and low-mass galaxies. Ignoring the contribution to the lensing signal between two halos, ${ }^{11}$ the lensing signal has three parts: the halo profile around central galaxies, the halo profile around satellite galaxies, and the central point source (i.e., the galaxy itself). For red galaxies, the higher amplitude of the $\Delta \Sigma$ measurements at scales $R \gtrsim 100 \mathrm{kpc}$ is indicative of the higher satellite fractions, as this scale probes the mass profile of the dark matter halo in its outskirts. Interior to this scale, the lensing signal is a measure of the mass of dark matter halos around central galaxies. For both bins in $M_{*}$ shown, the mean halo mass of centrals appears roughly consistent between passive and SF subsamples. The differences are driven primarily by the fraction of galaxies that are satellites.

\subsection{The Stellar-to-Halo Mass Ratios and their Evolution}

The left-hand panels in Figure 7 show the SHMR for red and SF galaxies at each redshift bin. The curves show the best-fit model for each sample, while the shaded regions indicate the range that contains inner $68 \%$ of the models. At low masses, the SHMR becomes shallow and stellar mass increases much more

\footnotetext{
11 The two-halo terms is included in all modeling, but has minimal impact on
} our results because we do not measure $\Delta \Sigma$ out past $1 \mathrm{Mpc}$. rapidly than halo mass: $M_{*} \sim M_{h}^{1 / \beta} \sim M_{h}^{2}$. As galaxy mass increases, however, the relation reaches a pivot point at which central galaxies increase in mass slower than their halos and the SHMR becomes steep. This is now accepted as a generic result of the abundance matching paradigm (Conroy et al. 2006; Wang et al. 2007; Conroy \& Wechsler 2009; Moster et al. 2010; Behroozi et al. 2010; Yang et al. 2012). In L12 we defined the pivot point quantitatively as the location in the $M_{*}-M_{h}$ relation were the $M_{*} / M_{h}$ ratio is maximal, usually around $M_{*} \sim 10^{10} M_{\odot}$ and $M_{h} \sim 10^{12} M_{\odot}$.

At all redshifts, the qualitative behavior of the SHMR for SF and passive galaxies is quite similar; both subsamples show a pivot point. The pivot halo mass is roughly $10^{12} M_{\odot}$ and the pivot stellar mass is roughly $10^{10.6} M_{\odot}$. We will present a more detailed comparison presently, but broadly speaking, there are few major differences in the results. When comparing the results at low masses, however, it is important to remember that these results do not reflect the fraction of halos occupied by red central galaxies. For the $z=0.66$ and $z=0.88$ redshift bins, the fraction of halos below $10^{12} M_{\odot}$ that have red central galaxies is vanishingly small. Only for $z=0.36$ does the red central fraction become significant at these halo mass scales.

At scales above the pivot point, however, the behavior of the SHMR is quantifiably different. At $z \sim 0.88$, massive SF galaxies occupy larger halos at fixed stellar mass. In each panel, the point with horizontal error bars shows the mean stellar mass within the X-ray group sample from George et al. (2011). Although the red central fraction from the groups is used within the MCMC chains, the mean stellar mass is not. At $z \sim 0.66$, massive SF galaxies still reside in more massive halos than their quiescent counterparts, but now the mean relations are much closer together. At $z \sim 0.36$, the mean SMHR for red and SF galaxies have crossed; massive passive galaxies 


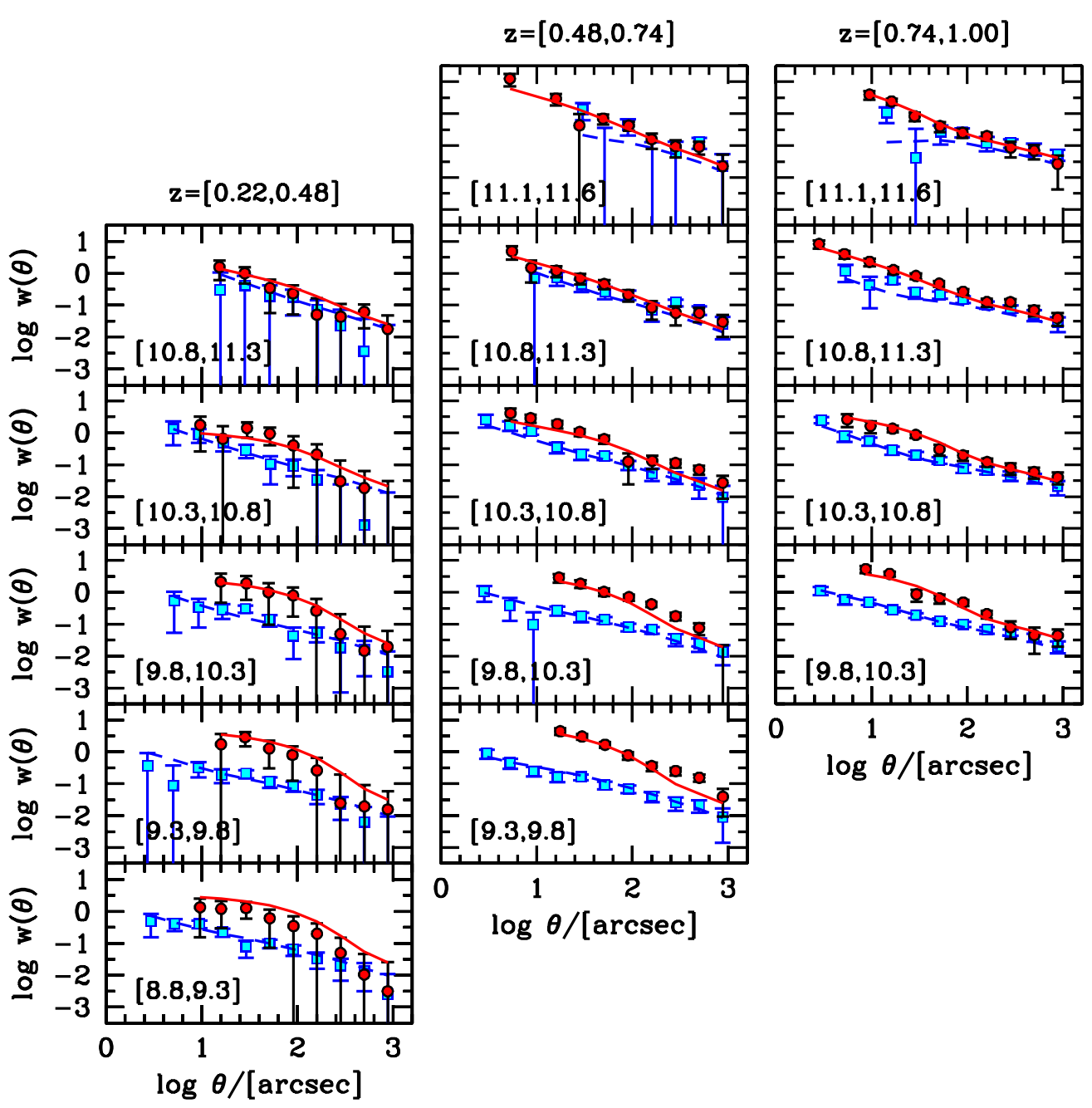

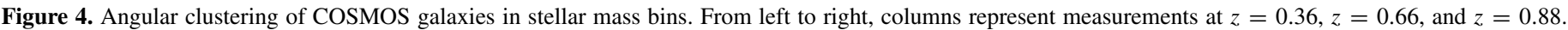

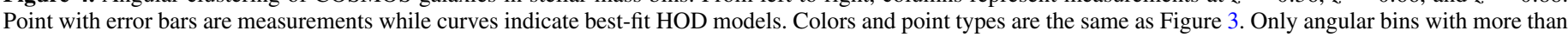

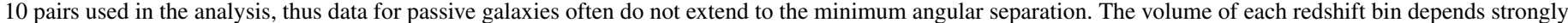

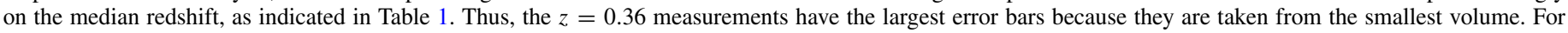
mass bins at $\log M_{*} \leqslant 10.3$, the enhanced clustering of passive galaxies is driven by the high fraction of satellite galaxies that are quenched (see Figure 11).

(A color version of this figure is available in the online journal.)

occupy slightly more massive halos than similar SF galaxies. The sample variance for the low $-z$ bin is significant, but an evolutionary trend can be seen across the full COSMOS sample. In T12 we compare these results to the central galaxies found in the group catalog, finding quantitative agreement. This figure also compares the new color-dependent results to the SHMR from L12. At low masses, the SF SHMR tracks the all-SHMR nearly exactly; this is expected given that SF galaxies dominate the population at these masses. At high masses, the all-SHMR is intermediate between the SF and passive SHMRs.

The origin of the differential evolution at the massive end comes from our specific combination of data. The SMFs clearly indicate that there are more passive galaxies than SF galaxies at the massive end of the spectrum. The clustering and lensing, however, indicate that the large-scale bias and halo masses of the SF and passive subsamples are consistent. Recall that the left-hand panels show the mean stellar mass as a function of halo mass, even though we have plotted the observable, $\log M_{*}$, on the $x$-axis. At fixed $M_{*}$, scatter becomes very important at the massive end. The right-hand panels in Figure 7 show the mean halo mass at fixed stellar mass. In this plot, the differences between the red and SF subsamples is almost entirely gone; thus, in bins of $M_{*}$ where satellite galaxies are negligible (i.e., at stellar masses significantly above the knee in the SMF), one would expect the clustering and lensing of SF and passive galaxies to be consistent. The difference in the SHMRs is driven by the larger values of $\sigma_{\log M_{*}}$ for SF galaxies than for passive galaxies. For $\mathrm{SF}$ galaxies at $z=0.88, \sigma_{\log M_{*}}=0.25 \pm 0.01$, while for passive galaxies $\sigma_{\log M_{*}}=0.18 \pm 0.05$. By $z=0.36$, the passive galaxies have the smaller scatter, and the steeper SHMR at the massive end. Although our functional form for $f_{\text {SHMR }}$ is meant to have a high degree of flexibility at high halo masses, we cannot rule out a possible bias due to our parametric form for $f_{\text {SHMR }}$. Additionally, the assumption of a symmetric, log-normal scatter may come into play in this regime where the scatter is important. With the current data we are unable to test alternative models for scatter.

To determine the origin of the constraints on the high-mass end of the SHMR, we ran a series of chains removing different data sets. Figure 8 show highlights from this series for the $z=0.88$ redshift bin. Intriguingly, the constraints when using the SMFs only already show clear indication of a separation between the SHMR of SF and passive galaxies, although the difference is not as large as the final result. Adding just the 

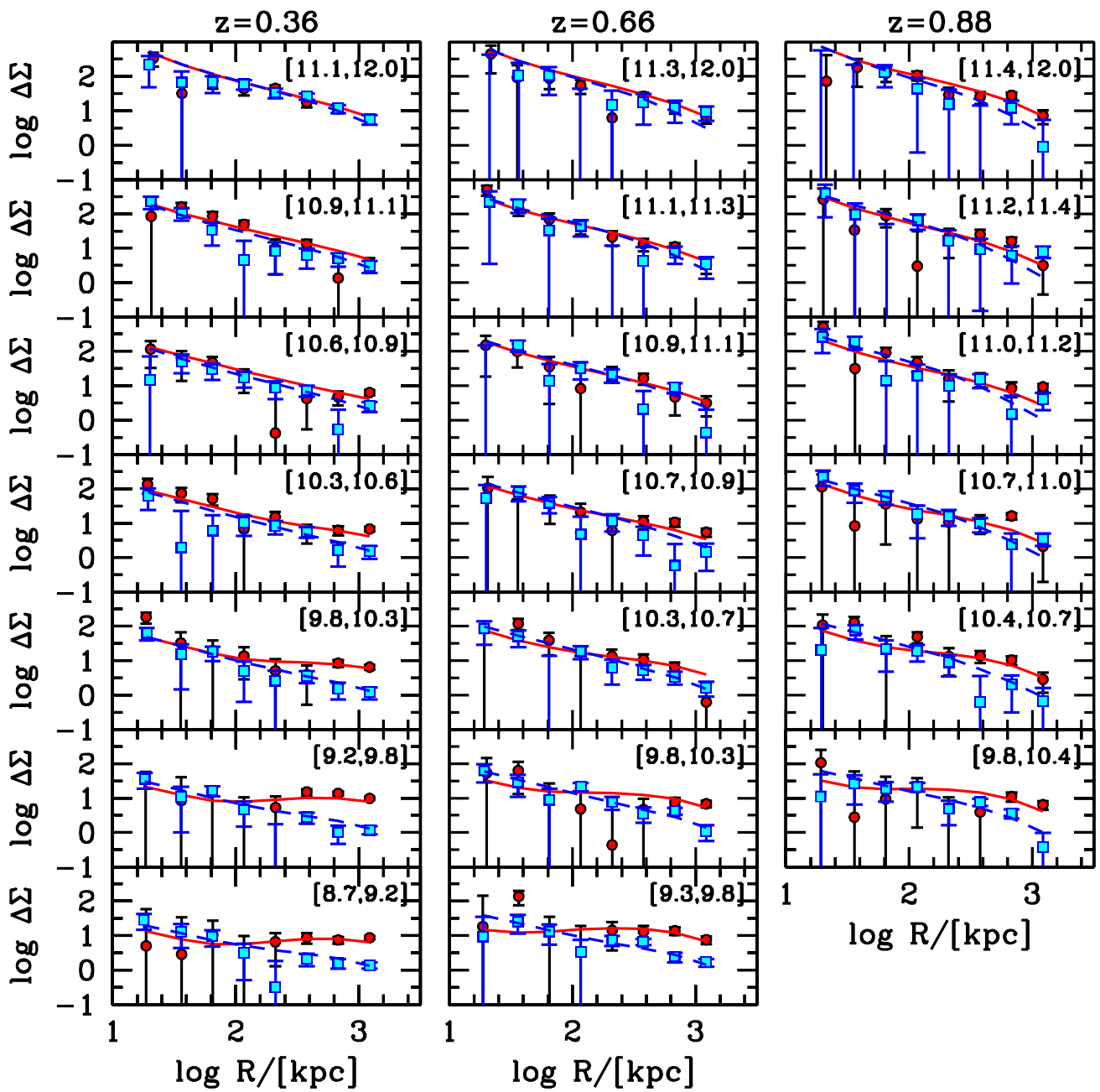

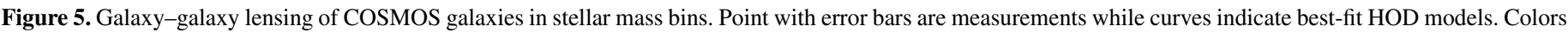

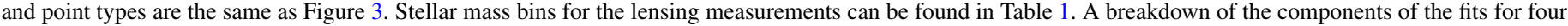
examples can be found in Figure 6.

(A color version of this figure is available in the online journal.)

most massive clustering bin increases the separation between passive and SF SHMR values into rough agreement with the full data. Similar results are found when removing the most massive clustering bin and incorporating all others; constraints on $\sigma_{\log M_{*}}$ come from a range of stellar masses, provided the halos occupied are in the regime where halo bias is monotonically increasing with halo mass (roughly $M_{h} \sim 2 \times 10^{11} M_{\odot}$ at this redshift). Because the halo bias function is highly non-linear, the mean halo mass is not the same as the bias-weighted halo mass. In this respect, the clustering has more constraining power on $\sigma_{\log M_{*}}$ than the lensing data. The top panel in Figure 8 demonstrates that our final results are not sensitive to the data derived from the X-ray groups. Results when removing the lensing data are similar.

\subsection{Central Red Fraction versus Halo Mass}

Figure 9 shows the $68 \%$ ranges of $f_{q}\left(M_{h}\right)$ from the MCMC chains for each redshift bin. At $z=0.66$ and $z=0.88, f_{q}\left(M_{h}\right)$ has a sharp cutoff between $M_{h}=10^{11.5-12.0} M_{\odot}$. Although the median value of the cutoff evolves to somewhat lower mass between $z=0.88$ and $z=0.66$, the results from the two redshift bins are also consistent with no evolution. At $z=0.36, f_{q}\left(M_{h}\right)$ is higher at all halo masses, most notably at $M_{h} \lesssim 10^{11.5} M_{\odot}$; rather than a sharp cutoff in the quenched central fraction, there is a long tail toward lower masses where the $f_{q}\left(M_{h}\right)$ is $3 \%-10 \%$. This is driven by all three sets of data: a higher abundance of lowmass passive galaxies in the SMF, lower clustering amplitude at for low-mass samples in $w_{\theta}$ and a lower satellite fraction in the $\Delta \Sigma$ measurements. We will explore this in detail in subsequent sections.

Figure 9 also shows results from the $z=0$ SDSS groups catalog of Tinker et al. (2011). The shape of $f_{q}\left(M_{h}\right)$ from the groups is similar to our non-parametric fit in COSMOS, but the amplitude is higher by $\sim 0.1-0.2 \mathrm{dex}$. This may reflect evolution given that the time elapsed between $z=0.36$ and $z=0.05$ is $3.3 \mathrm{Gyr}$, equal to the time elapsed from $z=0.88$ to $z=0.36$. It may also reflect differences in the definition of "quenched"; in Tinker et al. (2011), a $4000 \AA$ break below 1.6 is used to denote quenched, as opposed to NUV-optical-NIR colors cuts used on the COSMOS data. Although this definition is less sensitive to dust than the traditional $g-r$ color, $D_{n} 4000$ may suffer from aperture bias for more massive galaxies. The results for COSMOS groups are plotted as well, one datum per redshift bin, color-coordinated with the MCMC results.

Tinker \& Wetzel (2010) constrained halo occupation for color-selected clustering from DEEP2 and COMBO17, concluding that there was not a strong cutoff in $f_{q}\left(M_{h}\right)$ (additional 

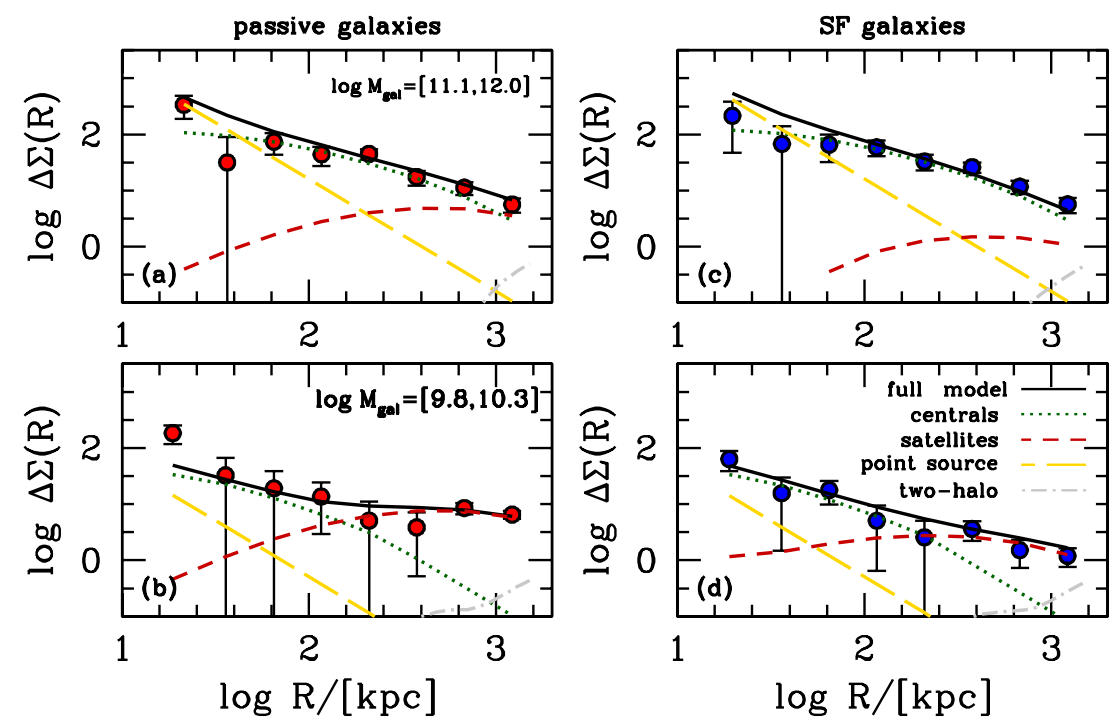

Figure 6. Breakdown of the lensing fits for two stellar mass bins in the $z=0.36$ redshift bin. The top row (panels (a) and (c)) show the most massive galaxy bin for passive and SF galaxies. The bottom row shows the $[9.8,10.3]$ stellar mass bin. The solid curve (black) shows the overall fit, which is the sum of the other curves. The dotted curve (green) is the lensing profile of the dark matter halo around central galaxies. The short-dash curve (red) shows the lensing profile of the halos around satellite galaxies. The long-dash (yellow) curve represents he central point-source-i.e., the central galaxy itself. The dash-dot (gray) curve is the lensing contribution from nearby halos-i.e., the two-halo term. For both mass bins, the passive galaxies have a higher fraction of satellites, evinced by the higher amplitude of the satellite lensing signal. At fixed $M_{*}$, the halos that host the central galaxies are roughly equal mass between passive and SF galaxies (this does not mean that $f_{\mathrm{SHMR}}$ is the same-we will discuss the differences between $\left\langle M_{*} \mid M_{h}\right\rangle$ and $\left\langle M_{h} \mid M_{*}\right\rangle$ in the following section).

(A color version of this figure is available in the online journal.)
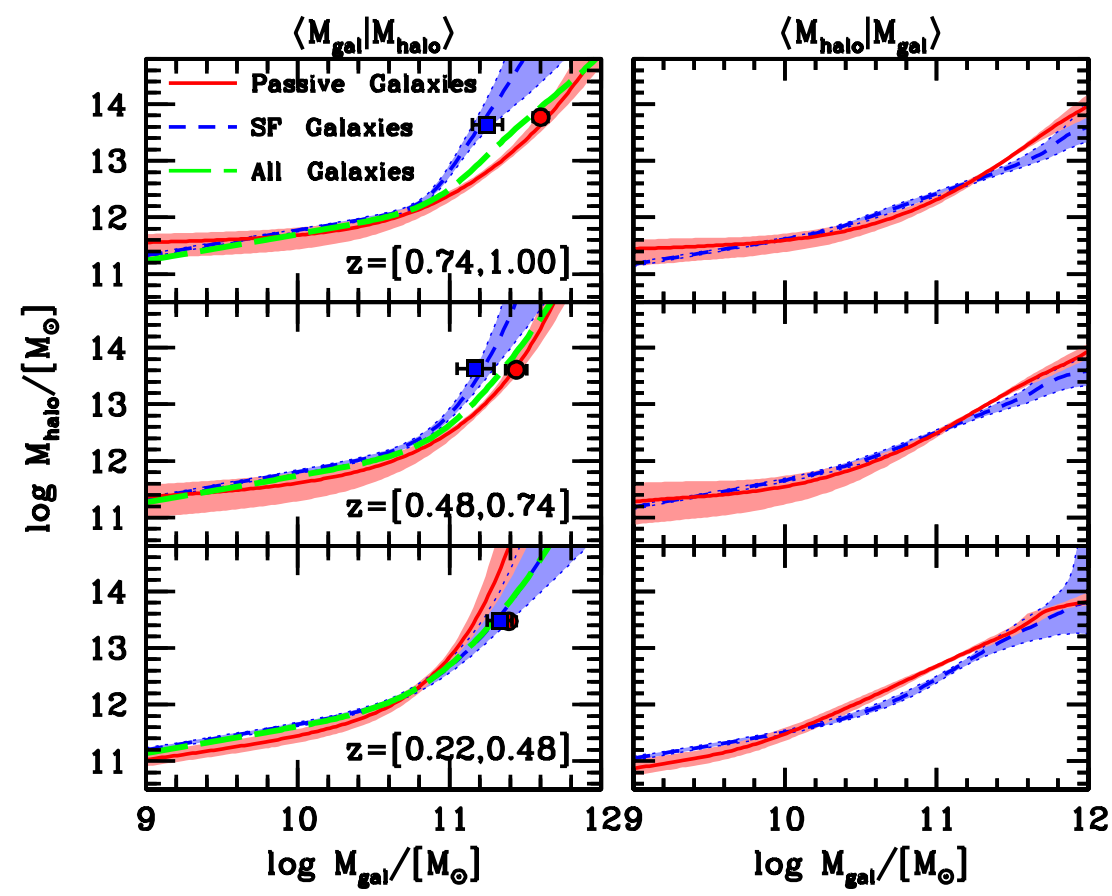

Figure 7. Stellar-to-halo mass ratios for passive and SF central galaxies. Shaded regions indicate $68 \%$ range in each quantity from the MCMC chains. The left-hand panels show $f_{\mathrm{SHMR}}$ for each redshift bin, equivalent to the mean $M_{*}$ at fixed $M_{h}$. The points with horizontal error bars represent the mean halo masses of the X-ray groups with passive and SF central galaxies, taken from T12. Long dashed curves show the SHMR for all galaxies, taken from L12. The right-hand panels show $\left\langle M_{h} \mid M_{*}\right\rangle$. The larger scatter for SF galaxies creates more Eddington bias, thus when binned in $M_{*}$, the mean halo mass is significantly smaller than $f_{\mathrm{SHMR}}$. Thus the lensing signals for massive galaxies are similar between passive and SF samples.

(A color version of this figure is available in the online journal.)

data from the UKIDSS-UDS were inconclusive). The clustering samples were created using a single color cut without any NIR data, contaminating the red sequence with dust-reddened SF galaxies. From Figure 9, many of these galaxies are centrals in low-mass halos, making $f_{q}\left(M_{h}\right)$ appear flatter and without any strong cutoff. Zhu et al. (2011) find that $\sim 25 \%$ of sub- $L^{*}$ galaxies with red colors are SF with specific rates of $\sim 10^{-10} \mathrm{yr}^{-1}$.
We note again that the detailed constraints on $f_{q}\left(M_{h}\right)$ depend on our assumption that quenching of central galaxies is a function of halo mass, independent of stellar mass. Because the mean galaxy mass at fixed halo mass is similar between passive and SF subsamples, a parameterization of $f_{q}$ that depends on stellar mass rather than halo mass will likely yield consistent results. 


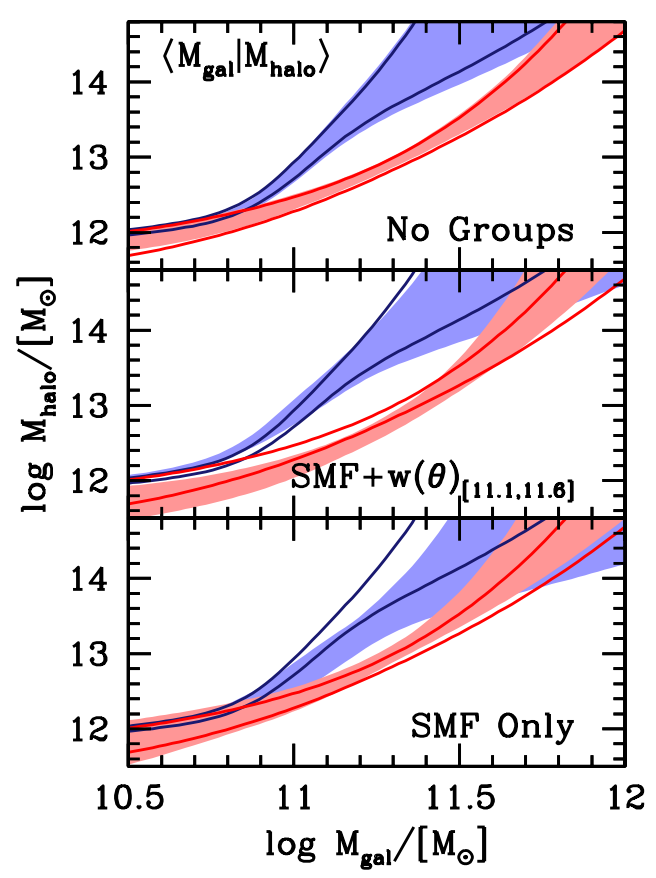

Figure 8. Stellar-to-halo mass ratios for passive and SF central galaxies when using only subsets of the available data. In each panel, the shaded region is the $68 \%$ confidence interval for the SHMR from the MCMC chains. The lines indicate the same quantity from the original chains using all data (see Figure 7). All panels show results from the $z=0.88$ redshift bin. Bottom panel: chains that incorporate only the stellar mass functions and their ratio. Middle panel: the stellar mass functions, the SMF ratio, and the most massive clustering bin: galaxies with $\log M_{*}=[11.1,11.6]$. Top panel: chains using all data except $f_{q}\left(M_{h}\right)$ from the X-ray group catalog.

(A color version of this figure is available in the online journal.)

\subsection{Central-Satellite Decomposition of the Stellar Mass Functions}

Figure 10 shows the SMFs for the SF and passive subsamples, broken down into the separate abundances of central and satellite galaxies. For SF galaxies, there is a modest increase in the number of both central and satellite galaxies in time. The abundance of red satellite galaxies exhibits little redshift evolution at low masses. There is actually a deficit of massive red satellites at $z=0.36$. It is unclear whether this represents physical evolution versus sample variance, an issue we will discuss in this subsection.

The only subsample that exhibits significant redshift evolution is red central galaxies. At the massive end there is minimal evolution, consistent with larger surveys results of the evolution of the luminosity function of luminous passive galaxies (Cool et al. 2008; Wake et al. 2006). However, at $M_{*} \lesssim 10^{11} M_{\odot}$, the number of red central galaxies increases rapidly from $z=0.88$ to $z=0.36$. At $M_{*}=10^{10} M_{\odot}$, this abundance increases by 1.2 dex. At this same mass scale, the change in the number of satellite galaxies is negligible.

This result is more clearly expressed by looking at the fraction of galaxies that are red, and how this fraction depends on categorization as a central or a satellite galaxy. Figure 11 shows $f_{q}$ as a function of redshift for five values of $M_{*}$ over the range $\log M_{*}=[9.7,11.2]$. In this figure we have included data from the SDSS groups catalog of Tinker et al. (2011). The differences in stellar mass estimates between the two surveys make comparisons of absolute abundances problematic, but fractions are more robust. To create the SDSS data in this figure, we

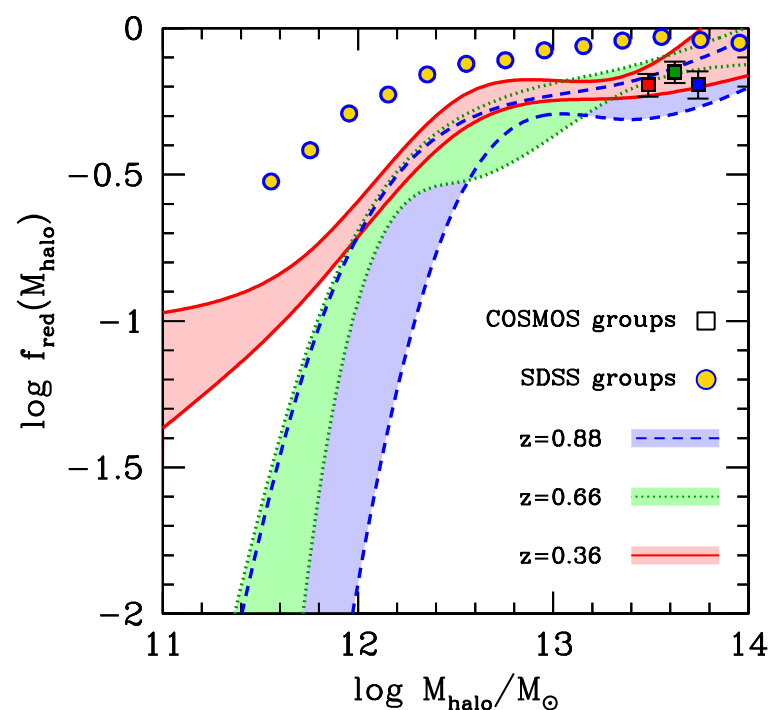

Figure 9. Fraction of central galaxies that are red as a function of halo mass, $f_{q}\left(M_{h}\right)$, for all three redshift slices. The shaded regions show the $68 \%$ range of values within the MCMC chains. The filled circles show the same quantity for the SDSS groups catalog of Tinker et al. (2011). The filled squares show the quenched fraction of central galaxies in COSMOS groups (George et al. 2011) that are used in the MCMC modeling. At $z \geqslant 0.66$, there is a sharp cutoff in $f_{q}\left(M_{h}\right)$, implying that nearly all central galaxies at $M_{*} \lesssim 10^{10.5} M_{\odot}$ are star forming at these redshifts. At lower redshifts, this cutoff moves to lower halo masses and there is a non-negligible contribution to the red sequence from low-mass central galaxies.

(A color version of this figure is available in the online journal.)

have added 0.2 dex to the stellar mass estimates and added 0.2 dex of scatter. The former represents the 0.2 dex shift in the SMFs between SDSS (Li \& White 2009) and COSMOS once deconvolved to a common scatter value. The latter is meant to mock up the increase uncertainties between SDSS spectroscopic redshifts and COSMOS photometric redshifts (see the discussion in Figure 14 in L12). Both of these changes lower $f_{\mathrm{Q}}$ by 0.1 to 0.2 , which the shift in mass scale dominating the effect. The upper error bars show the original SDSS values before shifting and adding scatter. Because both alterations to the SDSS data lower $f_{\mathrm{Q}}$, the values used here should be considered lower limits on the quenched fraction of SDSS galaxies.

Figure 11(a) shows $f_{q}$ for all galaxies in each stellar mass bin. The rate of change in $f_{q}$ with redshift monotonically decreases with increasing stellar mass. For massive galaxies, $f_{q}$ is roughly constant. At $\log M_{*}=9.7, f_{q}$ increases by a factor of five. Figure 11(b) shows the same quantity, but now for satellite galaxies only. Aside from the lowest mass bin, $f_{q}$ (sat) in all bins is consistent with no redshift evolution. Central galaxies, on the other hand, show significant evolution; at $\log M_{*} \lesssim 10, f_{q}$ (cen) increases by an order of magnitude. Even at $\log M_{*}=10.5$, $f_{q}$ (cen) increases by a factor of five over our redshift baseline.

Knobel et al. (2013) use group catalogs in the zCOSMOS survey to measure the redshift evolution of centrals and satellites as well. Due to the flux limit of the zCOSMOS target selection, they only achieve a redshift baseline for galaxies $M_{*} \gtrsim$ $10^{10.3} M_{\odot}$. They also find little to no evolution of the red fraction of satellites. For central galaxies, however, they find weaker evolution for the red fraction of central galaxies. In Figure 12 we compare $f_{q}$ for centrals and satellites between the two methods. An objective comparison is obstructed by the overall offset in $f_{q}$ between the two definitions of quenched (see Figure 2). Both approaches yield a small decrease in $f_{q}$ (sat) 

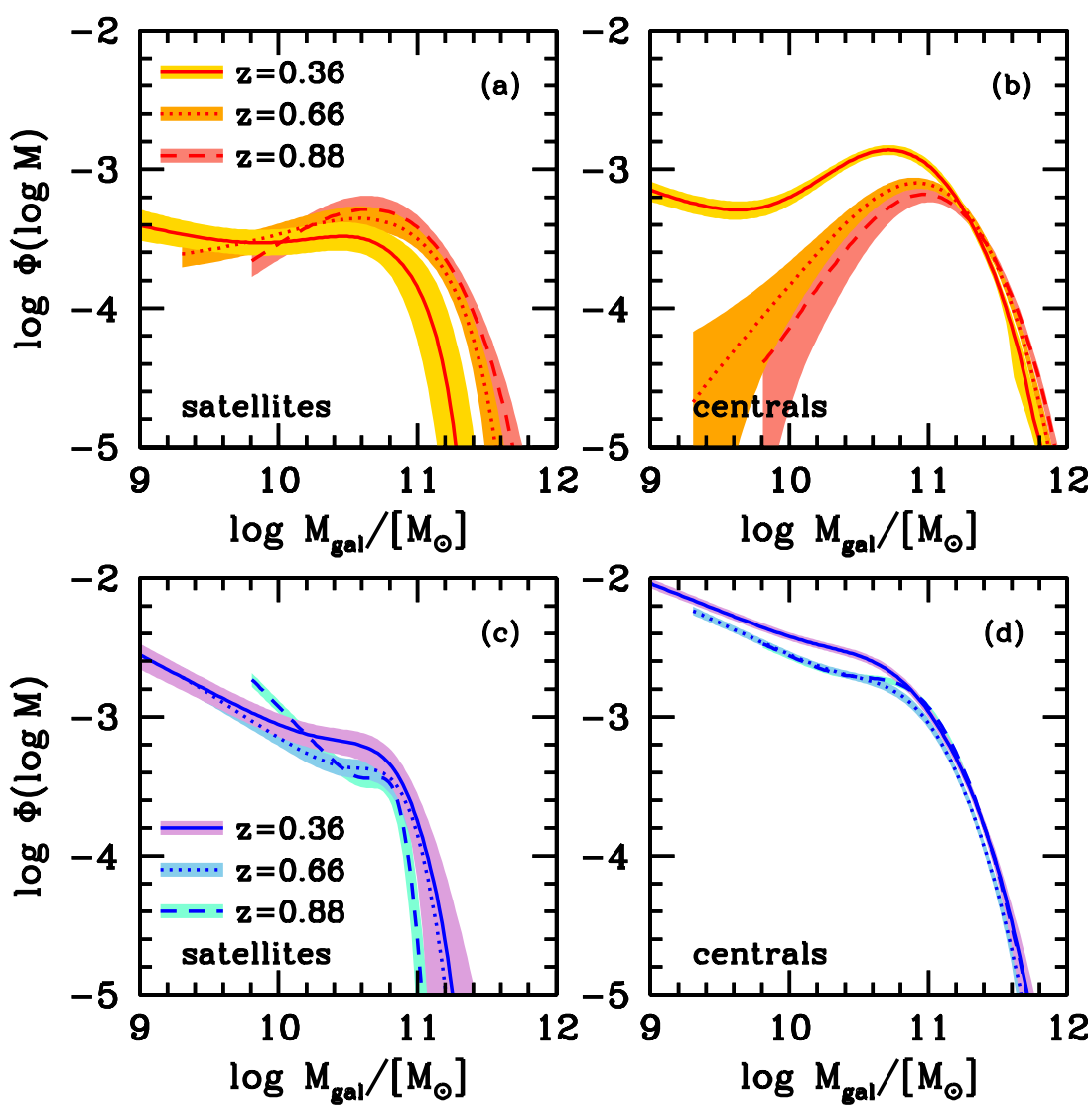

Figure 10. Stellar mass functions of passive and SF cosmos galaxies broken down into the contributions from central and satellite galaxies. Panels (a) and (b) show results for quenched satellite and central galaxies, respectively. Panels (c) and (d) show results for star-forming satellite and central galaxies, respectively. The shaded regions represent the $68 \%$ range of values within the MCMC chains. For all four subsamples, there is little redshift evolution at the high-mass end $\left(M_{*} \gtrsim 10^{11} M_{\odot}\right)$. There is a dearth of high-mass quenched satellites at $z=0.36$, but this is likely a statistical outlier. At low masses, the only subsample that shows significant evolution is passive central galaxies; at $M_{*}=10^{10} M_{\odot}$, the abundance of passive red centrals increases by more than an order of magnitude across our redshift baseline. Figure 11 shows that this growth in the fraction of quenched central galaxies continues to increases to $z=0$.

(A color version of this figure is available in the online journal.)
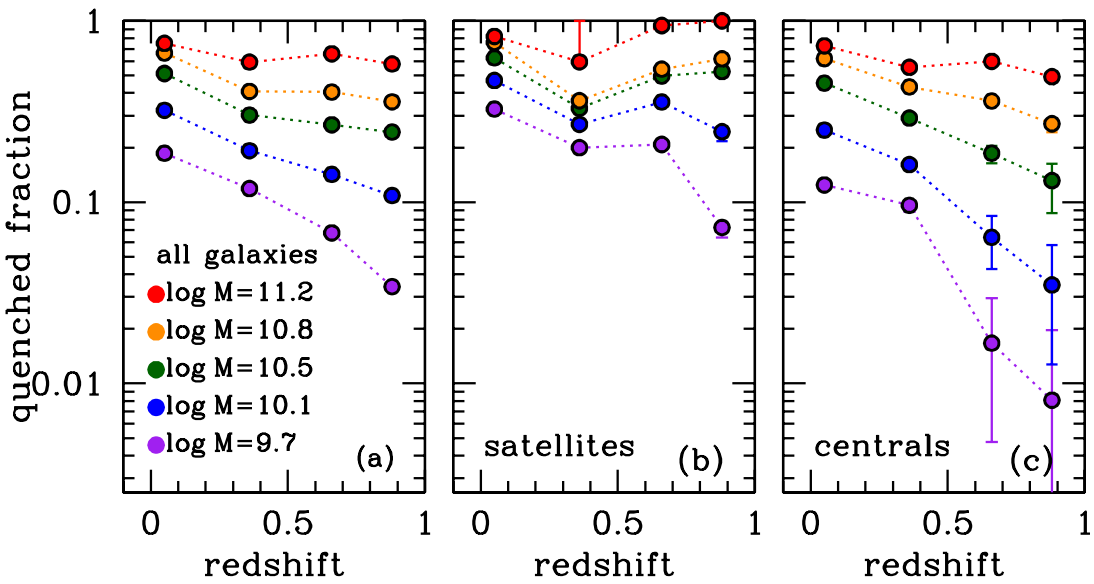

Figure 11. Red (quenched) fraction of galaxies as a function of redshift for various stellar mass bins. Panel (a) shows $f_{q}$ for all galaxies. Panel (b) shows $f_{q}$ for satellite galaxies. Panel (c) shows $f_{q}$ for central galaxies. Error bars on the COSMOS measurements represent the $68 \%$ range within the MCMC chains. Data points at $z=0.05$ are from the SDSS groups catalog of Tinker et al. (2011). The SDSS stellar masses have been modified to afford better comparison to COSMOS stellar masses, but these changes yield little to no change in the values on the $y$-axis. See text for details. Although the $z=0.36$ redshift bin is somewhat anomalous in its statistics, it is consistent with the overall trends in this figure: namely, the monotonic growth of the quenched fraction of galaxies at all masses, the near-constant quenched fraction of satellite galaxies, and the rapid growth of a population of quenched central galaxies, especially at low masses.

(A color version of this figure is available in the online journal.)

for massive galaxies as $z$ decreases, but the Knobel et al. (2013) groups yield a quenched fraction at $M_{*}=10^{11} M_{\odot}$ that is nearly unity. In this panel we plot the results from the COSMOS X-ray groups of George et al. (2011), which use the same definition of quenched as this work. The $f_{q}$ (sat) values are mostly consistent with those from our SHMR analysis. For central galaxies, the Knobel et al. (2013) groups yield contrasting results above and below $M_{*}=10^{10.5} M_{\odot}$. Below this limit, the zCOSMOS 

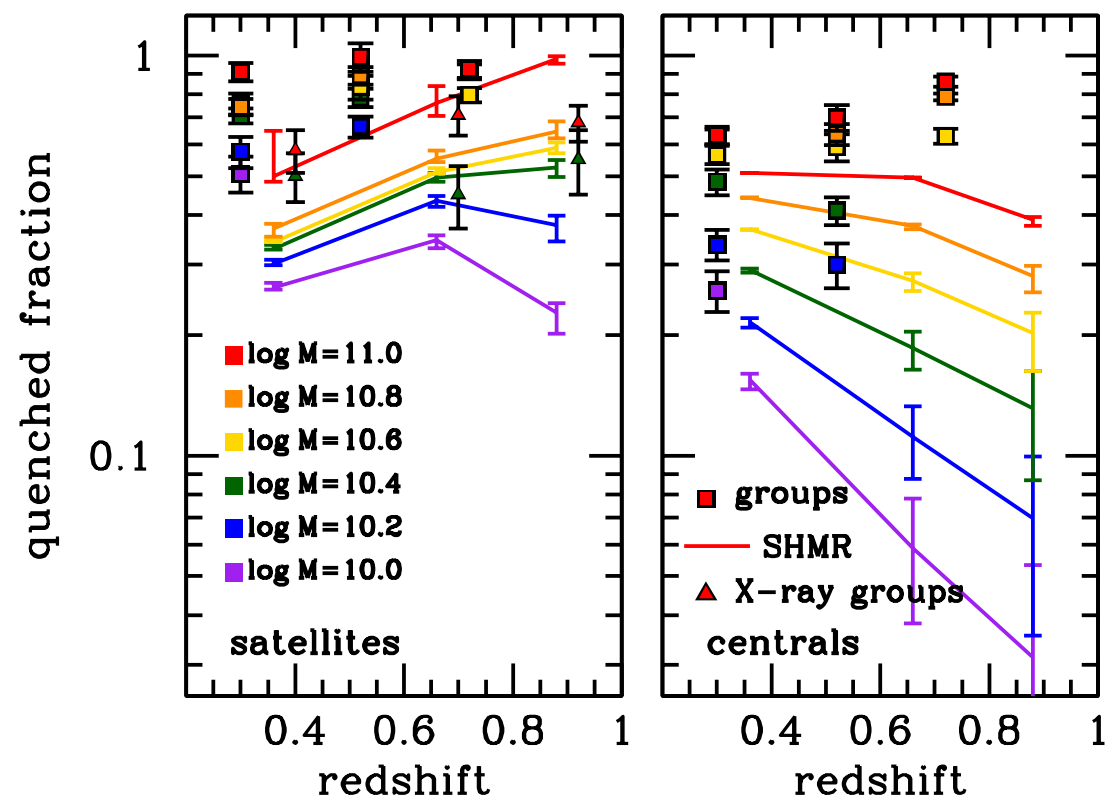

Figure 12. Evolution of the quenched fraction from our SHMR analysis compared to that from the zCOSMOS groups catalog of Knobel et al. (2013). The color scheme is the same as previous figures, but here we make the comparison in the stellar mass bins used in Knobel et al. (2013). The left panel shows $f_{\mathrm{Q}}$ for satellites while the right panel shows $f_{\mathrm{Q}}$ for centrals. In the left panel, we also include results from the COSMOS X-ray group catalog of George et al. (2013). There is an overall shift in the total quenched fractions for the COSMOS and zCOSMOS samples (see Figure 2) such that the zCOSMOS sample has a higher fraction of quenched galaxies. For massive objects, the zCOSMOS sample has a decreasing $f_{q}$ with decreasing $z$, which is driven by the decrease in $f_{q}$ (cen) for bins at $M_{*}>10^{10.5} M_{\odot}$. The photometric COSMOS sample used here has a monotonically increasing (or constant) overall red fraction, also driven by the behavior of the central galaxies. (A color version of this figure is available in the online journal.)
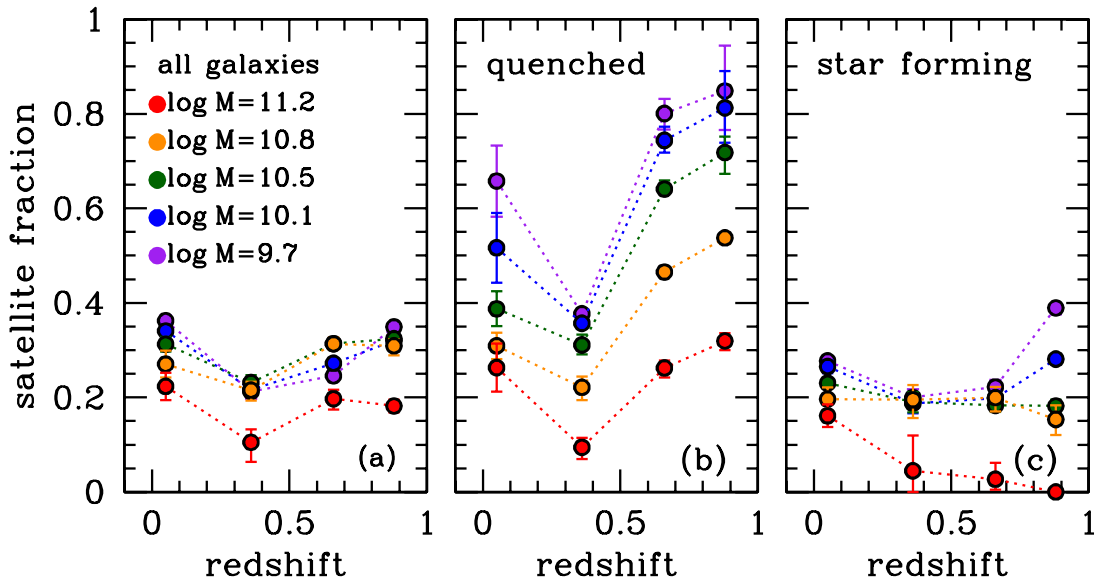

Figure 13. Satellite fraction of galaxies as a function of redshift for various stellar mass bins. Panel (a) shows $f_{\text {sat }}$ for all galaxies. Panel (b) shows $f_{\text {sat }}$ for red galaxies. Panel (c) shows $f_{\text {sat }}$ for star-forming galaxies. Error bars on the COSMOS measurements represent the $68 \%$ range within the MCMC chains. Data points at $z=0.05$ are from the SDSS groups catalog of Tinker et al. (2011). The SDSS stellar masses have been modified to afford better comparison to COSMOS stellar masses, but these changes yield little to no change in the values on the $y$-axis. See text for details.

(A color version of this figure is available in the online journal.)

central galaxies show a moderate increase in $f_{q}$ (cen), but above this limit the zCOSMOS central galaxies exhibit significantly decreasing $f_{q}$ (cen) with decreasing redshift. The quenched fraction of $M_{*}=10^{11} M_{\odot}$ centrals decreases from $90 \%$ to $60 \%$ over their redshift baseline.

The $U-B$ color cut used in zCOSMOS may be susceptible to dust contamination, which may be stronger at higher redshifts where star formation rates are also higher. Additionally, there may be differences driven by the two methods-halo occupation and group finding. Misclassification of which galaxy in a group is the central is a major source of bias for group catalogs (Skibba et al. 2011). Given that the quenched fraction of satellites exhibits no redshift evolution, this type of bias will only weaken the true trend of $f_{q}$ (cen). Moreover, Knobel et al. (2013) use a probabilistic scheme to select subsamples of centrals and satellite galaxies that have purity near $80 \%$, forcing them to assume that these subsets are representative of the overall populations. Halo occupation methods do not suffer from these biases, as central and satellite populations are constrained only in a statistical fashion, and not on an object-by-object basis. We also note that the central galaxies in the X-ray group catalog used here are a much cleaner sample of central galaxies, given that the group center can be verified with the X-ray brightness profile.

Figure 13 shows a complementary statistic: the fraction of galaxies that are satellites, $f_{\text {sat }}$, for the same stellar mass bins and redshift range. For all galaxies, $f_{\text {sat }}$ is between $0.25-0.35$, consistent with previous analyses of $z=0$ luminosity-dependent 

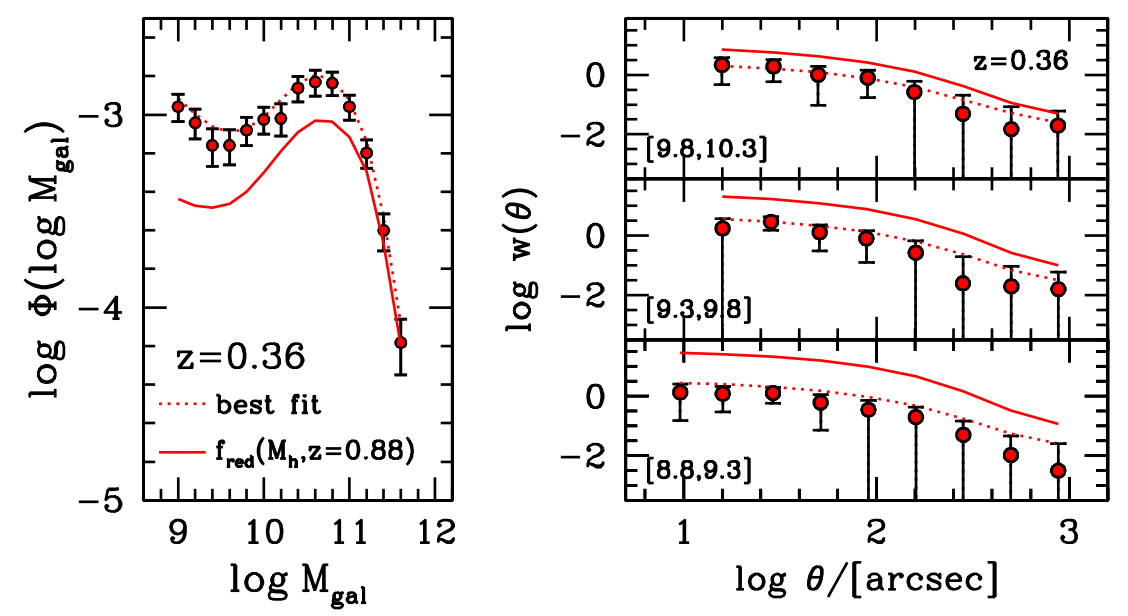

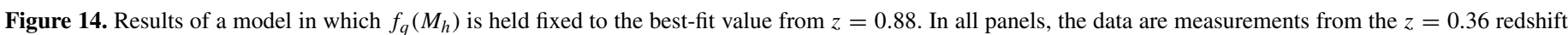

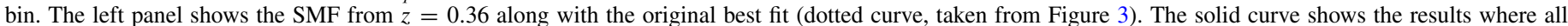

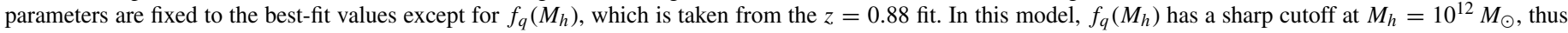

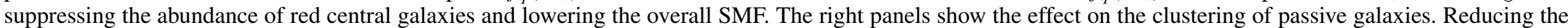

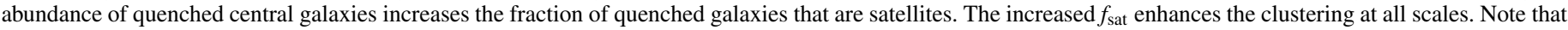

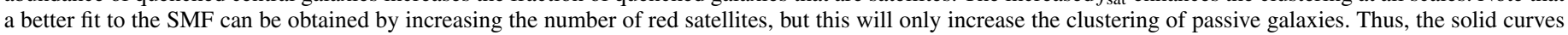
should be considered a lower limit on $w_{\theta}$ for models in which $f_{q}\left(M_{h}\right)$ does not evolve from $z=1$ to $z=0.2$.

(A color version of this figure is available in the online journal.)

clustering (e.g., Zehavi et al. 2005; Tinker et al. 2007; van den Bosch et al. 2007; Zheng et al. 2007; Zehavi et al. 2011). Halo occupation analysis of $z \sim 1$ luminosity dependent clustering indicates a somewhat smaller $f_{\text {sat }}$ than at $z=0$ (Zheng et al. 2007; Abbas et al. 2010). However, recent analysis of stellarmass dependent clustering at $z=1-2$ by Wake et al. (2011) find $f_{\text {sat }}$ values consistent with those in COSMOS. Due to the fact that satellite galaxies are predominantly red, they are fainter than SF galaxies at the same stellar mass, lowering the satellite fraction at a given mass. For SF galaxies, star formation rates increase with redshift, increasing the difference between luminosity and stellar mass defined samples.

The satellite fractions of SF galaxies are lower than for the full sample, generally near $\sim 0.2$, with minimal redshift evolution. Satellites dominate the population of low-mass passive galaxies at $z \sim 1$. Even at $\log M_{*}=10.8, f_{\text {sat }}=0.55$. By $z=0$, satellites represent less than half of passive galaxies at $\log M_{*}>$ 9.7. The change in $f_{\text {sat }}$ for passive galaxies is non-monotonic when incorporating the SDSS data, yielding a "dip" in $f_{\text {sat }}$ at $z=0.36$. The small volume of this redshift slice raises the possibility that the galaxy distribution around $z=0.36$ within COSMOS is a significant outlier with respect to the cosmic mean. We note that while the trend of $f_{\text {sat }}($ red $)$ with redshift is non-monotonic, the trend in $f_{q}$ (cen) is monotonic. Thus, if the $z=0.36$ redshift slice is simply removed from consideration, the results in Figures 11 and 13 are still consistent with the scenario in which the only population to undergo significant evolution since $z=1$ is red central galaxies.

\subsection{Signature of the Evolving Red Central Population in the Data}

Figure 14 demonstrates where our constraints on the evolving population of red centrals derive from. If we assume that the fraction of halos with red centrals is fixed at $z=0.88$, the abundance and clustering of the overall red population is markedly different at $z=0.36$. Figure 14 shows results from the HOD model at $z=0.36$, but the five parameters of the nonparametric $f_{q}\left(M_{h}\right)$ function have been replaced by the best-fit values at $z=0.88$. In this model, the abundance of low-mass is low by a factor of $\sim 2.5$ relative to the data, while the clustering is too high by an order of magnitude or more. The increased clustering amplitude is attributed to the higher satellite fraction of passive galaxies in this model. It is possible to construct a model with the $z=0.88 f_{q}\left(M_{h}\right)$ that relieves the tension with the SMF, but this requires making up the difference by increasing the number of satellite galaxies, which only increases the tension with the clustering. In short, the only way to match both the SMF and $w_{\theta}$ measurements at $z=0.36$ is to increase the frequency of quenched central galaxies relative to $z=0.88$.

\section{DISCUSSION}

In an upcoming paper we will present a detailed analysis of the halo occupation results presented here, comparing these results to the growth histories, merging rates, and subhalo accretion and evolution in high-resolution $N$-body simulations. But it is possible to make significant qualitative assessment of our breakdown of the red sequence into central and satellite galaxy components.

Evolution of SHMR for high-mass galaxies. Observations indicate that the red sequence begins with massive galaxies at $z \gtrsim 2$ (Kriek et al. 2008; Williams et al. 2009). Thus it is not surprising that massive passive and SF galaxies have substantially different SHMRs at $z=1$. Both the halo occupation analysis presented here and the X-ray groups in analyzed T12 indicate that, above the group halo mass scale $\left(\gtrsim 10^{13} M_{\odot}\right)$, SF central galaxies are less massive than their red counterparts at fixed $M_{h}$. The substantial difference between $M_{*}$ for SF and passive subsamples implies that star formation is not a stochastic process in these objects: if massive central galaxies underwent periodic episodes of star formation followed by longer-term quiescence, the galaxies at fixed halo mass would have the same stellar mass. The results also imply that massive quenched galaxies formed their stars very rapidly at high redshift, essentially getting "ahead of the growth curve" relative to central galaxies that would still be forming stars by $z=1$. At high redshift, central galaxies essentially "knew" they would be quenched by $z=1$ (see the discussion in T12). 
From $z=1$ to $z=0$, the SHMRs evolve quite differently depending on star formation activity. By $z=0.36$, the mean relations have crossed and red central galaxies live in higher mass halos than SF central galaxies at fixed mass. This inversion is also consistent with results from $z=0$ studies (Mandelbaum et al. 2006; More et al. 2011). SF galaxies of mass $10^{11} M_{\odot}$ grow by a factor of $\sim 1.6$ using the star formation rates of Noeske et al. (2007) from $z=0.88$ to $z=0.36$. Host halos for these galaxies $\left(M_{h} \sim 10^{13}\right)$ grow by a factor of $\sim 1.8$ over the same redshift interval (Wechsler et al. 2002), thus SF central galaxies grow almost as fast as their host halos. For quenched galaxies, their growth rates are significantly slower than that of their host halos, causing the inversion of the SHMR seen in Figure 7. Although halos will accrete substantial stellar mass from smaller galaxies, most of this mass does not merge with the central galaxy; this is implied by the evolution of the luminosity function of massive passive galaxies (Wake et al. 2006; Cool et al. 2008). This mass contributed to the buildup of the intracluster light (Conroy et al. 2007; Purcell et al. 2007). The results here will put strong constraints on the growth of massive passive galaxies in our follow-up paper.

Evolution of the SHMR for low-mass galaxies. In contrast to the massive end of the galaxy population, low-mass galaxies show little evolution in the SHMR as well as very little difference in the SHMR between passive and SF subsamples. Due to the low abundance of low-mass red central galaxies, the errors on the SHMR below the pivot point are much higher for passive galaxies relative to $\mathrm{SF}$ galaxies. For each redshift bin, the red SHMR is slightly below the SF relation (at fixed $M_{*}$ ), but they are consistent within the error bars. From Figures 10 and 11, the abundance of red centrals is nearly negligible at $z=1$ and increases rapidly relative to other constituents of the full galaxy population. Thus, most low-mass quiescent central galaxies will be recent additions to the red sequence and the halo masses of red galaxies will be similar to those of SF galaxies. Lowmass galaxies have significant gas content, with $M_{\text {gas }} \gtrsim M_{*}$ at $M_{*} \lesssim 9.5$ at $z=0$ (Baldry et al. 2008). The difference in stellar mass at fixed halo mass should be indicative of the amount of this gas that has been converted into stars during the quenching process. This proposition assumes no increase in the halo mass; i.e., the quenching mechanism is not major mergers.

The migration rate of central galaxies to the red sequence. Our results are in good agreement with recent measurements from PRIMUS from Moustakas et al. (2013) that demonstrate that the growth of the red sequence from $z=1$ to 0 is primarily due to low-mass galaxies being quenched of their star formation. Our SHMR analysis further indicates that this growth is happening in the low-mass central population as opposed to satellites in groups and clusters. Figure 15 shows the rate at which central galaxies are added to the red sequence. The $x$-axis is stellar mass and the $y$-axis is the difference in the abundance of red centrals between adjacent redshift bins, divided by the time lapse between redshift bins (units of number/volume/dex/ Gyr). The shaded regions shows the $1 \sigma$ range in rates given the uncertainties in the abundances in red centrals at each redshift. Within $1 \sigma$, there is evidence for an accelerated migration rate over the COSMOS redshift baseline, although the migration rates are consistent within their $2 \sigma$ uncertainties.

Figure 15 also shows the migration rate of central galaxies to the red sequence using the SDSS group catalog of Tinker et al. (2011). This group catalog allows us to isolate only the abundance of central quenched galaxies (quenched by the criterion of $\left.D_{n}(4000)>1.6\right)$. Although the redshift baseline

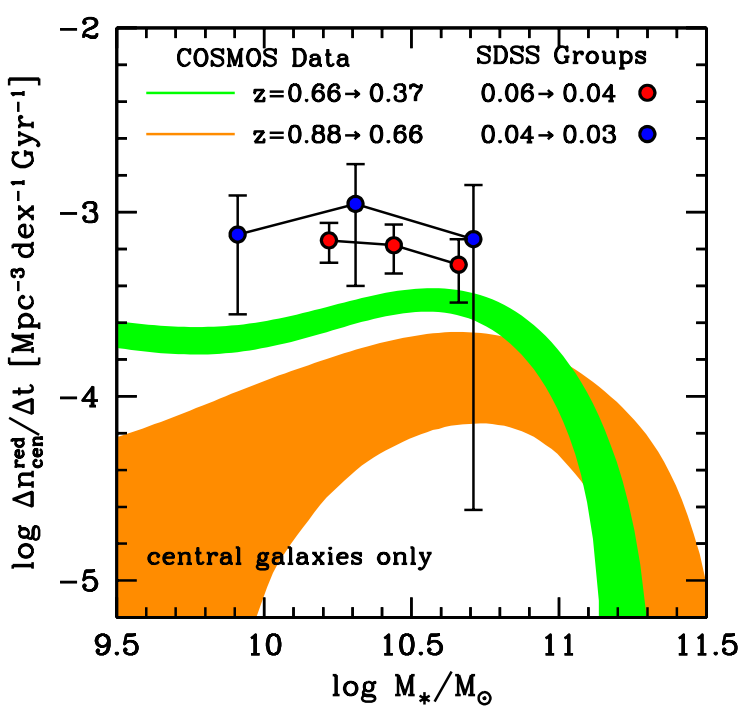

Figure 15. Rate at which central galaxies migrate to the red sequence as a function of stellar mass. The $y$-axis, $\Delta n / \Delta t$, represents the difference in the red central stellar mass functions between redshift bins, normalized by the time between each redshift. With three bins we are able to measure two values for $\Delta n / \Delta t$. The shaded regions show the $68 \%$ confidence intervals for this quantity after combining the uncertainties for each redshift bin. The points with errors represent the same quantity but using the sample of central galaxies from the SDSS group catalog. The red points use a volume-limited sample of groups complete to $M_{*}=10^{10.1} M_{\odot}$, with an upper redshift limit of 0.064 . The blue points use a volume-limited sample of groups complete to $M_{*}=10^{9.7}, M_{\odot}$, with a redshift limit of $z=0.04$. Error bars on both sets of points are Poisson. These results imply that the quenching efficiency for central galaxies at $M_{*} \lesssim 10^{10.5} M_{\odot}$ is increasing rapidly from $z=1$ to $z=0$.

(A color version of this figure is available in the online journal.)

within the SDSS Main galaxy sample is small, the overall number of galaxies is very large and it is possible to detect changes in the abundance of red central galaxies within the volume-limited group catalogs of Tinker et al. (2011; see their Table 1). As discussed earlier, direct comparison of the SDSS stellar masses with COSMOS stellar masses is not possible, but given the lack of significant slope of the migration rate with stellar mass, the difference in $M_{*}$ estimator is less relevant. The SDSS results yield a migration rate nearly an order of magnitude higher than the $z=0.88 \rightarrow 0.66$ COSMOS results.

Previous studies have also detected an acceleration of the migration rate onto the red sequence with cosmic time. Both the PRIMUS results and results from zCOSMOS of Pozzetti et al. (2010) find that growth rate, in number and mass density, for objects on the red sequence is increasing with decreasing redshift at $M_{*} \lesssim 10^{10.6} M_{\odot}$. These studies find significantly less evolution in the growth rate than in this work, which is a natural consequence of analyzing to overall galaxy population as opposed to focusing on central galaxies.

The results here make strong predictions for the minimum $M_{*}$ that can be quenched in the field. Geha et al. (2012) find that there are no isolated field galaxies below $10^{9} M_{\odot}$ that are passively evolving in the low-redshift NASA-Sloan Atlas. Our models predict that the $f_{q}$ (cen) drops below $1 \%$ at $M_{*}=3 \times 10^{9} M_{\odot}$ at $z=0.66$ and $M_{*}=6 \times 10^{9} M_{\odot}$ at $z=0.88$. Extending this search for the minimum quenched field galaxy can confirm and strengthen the constraints from the SHMR analysis.

The quenching timescale for satellite galaxies. The probability that a satellite is quenched increases monotonically with the time that has passed since it was accreted (Wetzel et al. 2013a); older satellites are more likely to be quenched of their star formation. Thus we can compare our constraints on $f_{q}$ (sat) with 


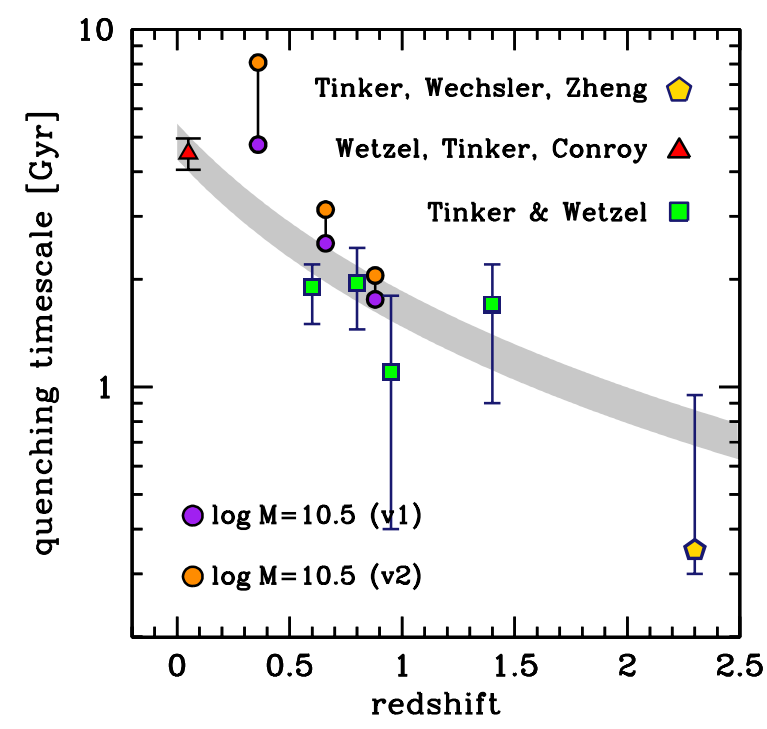

Figure 16. Quenching timescale of satellite galaxies as a function of redshift. The purple and orange filled circles show results for $M_{*}=10^{10.5} M_{\odot}$ galaxies in COSMOS. the "v1" method assumes that all galaxies were star-forming when accreted. The "v2" method uses $f_{q}$ (cen) from the redshift of the measurement to obtain $t_{\mathrm{Q}}$; these models bracket the physical range of models. The red triangle at $z=0.05$ is from the analysis of SDSS groups in Wetzel et al. (2013a), which models the evolution of the red central fraction explicitly. The green squares are taken from the clustering analysis of Tinker \& Wetzel (2010). In order of increasing redshift, these data points represent COMBO-17 (Phleps et al. 2006), DEEP2 $M_{B}<-19.5$, DEEP2 $M_{B}<-20.5$ (Coil et al. 2008), and UKIDSS-UDS (Williams et al. 2009). The yellow pentagon at $z=2.3$ is from Tinker et al. (2010b), analyzing the clustering of distant red galaxies from Quadri et al. (2008). The shaded band shows $t_{\mathrm{Q}} \sim(1+z)^{-3 / 2}$, normalized by the datum from the SDSS groups data. This power-law dependence on $z$ represents the change in the dynamical friction timescale as the mean density of halos changes proportionately with the mean density of the universe. The observations in COSMOS as well as the other sampled plotted above indicate that the fraction of red satellites is constant with redshift. Because satellite lifetimes decrease with increasing redshift, the quenching of satellite galaxies must be more efficient in the past.

(A color version of this figure is available in the online journal.)

our theoretical knowledge of the accretion and destruction of subhalos in $N$-body simulations. At high $z$, the mean age of a subhalo (i.e., the time that has elapsed since it was accreted) is significantly smaller than the mean age of subhalos at $z=0$. Dynamical friction is more efficient at higher redshifts because the mean density of dark matter halos increases as $(1+z)^{3}$. Making the ansatz that the oldest subhalos have the lowest star formation rates allows us to infer the timescale that must elapse for galaxies that are accreted as SF to migrate to the red sequence; e.g., if $50 \%$ of subhalos are older than 4 Gyr and $50 \%$ of satellite galaxies are red, it takes approximately 4 Gyr for satellite galaxies to be quenched of their star formation (Tinker et al. 2010b; Tinker \& Wetzel 2010; Wetzel et al. 2013a).

Figure 16 shows the estimated quenching time for $M_{*}=$ $10^{10.5} M_{\odot}$ satellite galaxies. Here we use the simulation results from Tinker \& Wetzel (2010). The two values represent the upper and lower bounds on the quenching timescale, based on assumptions about the fraction of satellite galaxies that were quenched prior to accretion; either that $f_{q}(\mathrm{cen})=0$ or that $f_{q}$ (cen) is the value at the redshift of the measurement. In reality, $f_{q}$ (cen) will be nonzero but lower than at the redshift of the measurement because galaxies were accreted at higher redshift. We compare these results to those for $M_{*}=10^{10.5} M_{\odot}$ galaxies $z=0$ (Wetzel et al. 2013a). This estimate takes into account the evolution in $f_{q}$ (cen). We also show results from Tinker et al. (2010b) and Tinker \& Wetzel (2010) at higher redshift. These latter papers analyze clustering for different luminosity-defined samples, so this is not an apples-to-apples comparison. But in general these results are consistent with a scenario in which the quenching timescale of satellite galaxies varies with the evolving dynamical timescale of the host halos: $t_{\mathrm{Q}} \sim(1+z)^{-3 / 2}$.

Peng et al. $(2010,2012)$ investigate the quenched fraction of galaxies as a function of local density, stellar mass, and redshift. They parameterize galaxy quenching as "mass quenching" and "environment quenching," demonstrating that the effects of these disparate mechanisms are fully separable. "Mass quenching" can be compared to central galaxy processes, while "environment quenching" is tightly associated with satellite processes. The fundamental difference in the approach of Peng et al. and this work is that the fundamental parameter in our approach is the mass of the host halo (and of the subhalo if it is a satellite), while Peng et al. consider the stellar mass to be fundamental for the central galaxies and local density of galaxies to be fundamental for the satellites. For central galaxies, due to the small scatter between stellar mass and halo mass, it may not be possible to distinguish between these two approaches. Further work is required to see if a model in which central galaxy quenching is determined by galaxy mass fits the data as well as the model we have presented here. It is worth noting that Peng et al. (2010) find their "mass-quenching efficiency" to increase with cosmic time, in agreement with our results.

For satellite galaxies, Peng et al. (2012) find that local density correlates better with quenching than either stellar mass of the satellite galaxy or host halo mass. This is at odds with our conclusions, as well as the model presented in Wetzel et al. (2012, 2013a, 2013b), in which the observed correlation between host halo mass and quenched fraction of satellite galaxies is driven by the time that has elapsed since the satellites were accreted. More massive halos have older subhalo populations, thus contain satellites that are more often quenched of their star formation. In the next paper in this series (A. Wetzel et al., in preparation), we model various physical mechanisms in detail, scrutinizing the local density model as a driver of satellite evolution.

Peng et al. (2010) find no evolution with redshift in their environment quenching efficiency, which is in stark contrast to the results in Figure 16 and our conclusion that satellite quenching efficiency is much higher in the past. The actual quenched fraction of satellite galaxies is nearly independent with redshift (see Figure 11 and Tinker \& Wetzel 2010), but Peng et al. (2010) do not take into account the redshift dependence of satellite dynamics discussed above, i.e., the fact that satellites at $z=1$ survive as satellites $\sim 1 / 3$ of the time $z=0$ satellites do. The Peng et al. (2010) results imply that the fraction of satellites that are quenched after accretion is time independent, thus their results are consistent with an efficiency (or timescale) that varies with the dynamical time of dark matter halos.

What is the mechanism responsible for the growth of the red sequence? The constant $f_{q}$ (sat) with redshift implies that the rates of creation and destruction of red satellite galaxies roughly balance. So although the mechanisms that quench star formation in groups and clusters-ram pressure, strangulation, harassment, etc.-are constantly acting on SF satellites to quench their star formation, they have minimal impact on change in the number of objects on the red sequence from $z=1$ to $z=0 .{ }^{12}$ The conclusion of Wetzel et al. 2013a is that roughly

\footnotetext{
12 We note that the mass of these "destroyed" satellites is not lost, but it is likely that much of it goes into intra-cluster light and is not accounted for by a simple mass-weighted integral over the red-galaxy stellar mass function.
} 
$1 / 3$ of $z=0$ quenched galaxies with $M_{*} \geqslant 10^{9.7} M_{\odot}$ were put on the red sequence by satellite-driven processes (their Figure 6). This is true whether averaging by number of galaxies or by total stellar mass. At $z=1$, this fraction was higher, but the overall number of objects on the red sequence was somewhat smaller. For central galaxies, the primary mechanisms proposed to quench star formation are AGN and major mergers, or perhaps a combination of the two as the latter may drive the former. To be in agreement with the results here, the mechanism for star formation quenching in central galaxies must satisfy two requirements: (1) become more efficient with time (i.e., as $z \rightarrow 0$ ) and (2) be roughly independent of stellar mass.

Let us take AGN and mergers as uncorrelated mechanisms. For mergers, Hopkins et al. (2010) find a general agreement among theoretical predictions and observational estimates in which the merger rate is $\sim 0.1 \mathrm{Gyr}^{-1}$ at $z=1$ and rapidly decreases by a factor of $\sim 5$ from $z=1-0$ for galaxies in the range $10^{10} M_{\odot}<M_{*}<10^{11} M_{\odot}$. There is also a strong stellar mass dependence on the major merger rate (Maller 2008; Stewart et al. 2009). Which mergers actually put galaxies on the red sequence is not fully quantified, given that merger simulations with gas-rich progenitors can yield SF disk galaxy remnants (Robertson et al. 2006; Hopkins et al. 2009). Regarding AGN, although theoretical models focus on AGN as a method to halt star formation in massive galaxies, observed SMFs of X-ray-AGN-hosting galaxies show little to no dependence on stellar mass (Bundy et al. 2008; Georgakakis et al. 2011). There is general consensus that AGN activity peaks at $z \approx 2$ and monotonically decreases toward $z=0$, but when quantified as a SMF of AGN hosts, the picture is less clear. Bundy et al. (2008) show no redshift evolution in the X-ray AGN host SMF over $z=[0.4,1.4]$, while Georgakakis et al. (2011) find a lower amplitude of this quantity at $z \approx 0$ relative to $z=1$. These results rely on the pencil-thin $0.5 \mathrm{deg}^{2}$ AEGIS field, so sample variance may be significant. As with galaxy mergers, connecting AGN to quenching requires knowledge of which AGN matter; is there an X-ray luminosity threshold for quenching? If so, does it depend on stellar mass or gas mass or redshift?

Another possibility is simply a lack of fuel for star formation. Behroozi et al. (2013) demonstrate that the overall mass accretion rate monotonically declines for all dark matter halos at $z \rightarrow 0$. If baryonic accretion falls accordingly, SF central galaxies may not have a high enough surface density of gas to continue forming stars.

Galaxy morphology affords an extra lever-arm in constraining power that we have not utilized in this paper. Bundy et al. (2010) find a population of passive disks at $z \sim 0.6$ but a paucity of such objects at lower $z$ (see George et al. 2013 for an investigation of such galaxies within groups). At low stellar masses, where we find the most significant increase in the red sequence, the morphological type with the highest fractional increase is ellipticals/S0, implying that the path the red sequence for low-mass central galaxies is accompanied by morphological change as well.

\section{SUMMARY}

We have constrained the stellar to halo mass relations for passive and SF galaxies over the redshift range $z=[0.2,1.0]$ in the COSMOS field. These constraints are derived from measurements of the SMF, the angular correlation function, and galaxy-galaxy lensing for multiple stellar mass bins within each redshift bin. For massive galaxies, $M_{*} \gtrsim 10^{10.6} M_{\odot}$, the SHMRs for passive and SF samples exhibit significant differential evolution, with passive galaxies growing much slower than their halos while SF galaxies grow roughly at the same rate as their host halos. At lower masses, there is little difference, implying that most faint passive galaxies are recent additions to the red sequence.

Our analysis affords a breakdown of the COSMOS galaxy population into central and satellite galaxies. With this breakdown, we demonstrate that the number of passive satellite galaxies shows little to no evolution with time, thus the change in the red sequence is driven by quenching of central galaxies, primarily at low masses. The overall migration rate of central galaxies to the red sequence is increasing with cosmic time, with the rate at $z=0.05$ being nearly a factor of 10 higher than that derived at $z=0.78$. Over the same redshift span, the quenching efficiency of satellite galaxies is decreasing with cosmic time. At $z=0.05$, the timescale for quenching is $\sim 2.5$ times longer than the quenching timescale for satellites at $z=0.88$.

We parameterize the quenching of central galaxies as being a function of their host halo mass. At $z=0.88$, we find a sharp cutoff in quenched central galaxies at $M_{h} \sim 10^{12} M_{\odot}$, a cutoff that shifts down $0.2-0.4$ dex by $z=0.66$. These results are reminiscent of recent theoretical work demonstrating a critical halo mass scale for shock-heating of infalling gas: the coldmode/hot-mode accretion scenario (e.g., Birnboim \& Dekel 2003; Kereš et al. 2005, 2009; Dekel \& Birnboim 2006). This shift continues to $z=0.36$, but at this redshift there is also a tail of quenched central galaxies that extend to $M_{h} \lesssim 10^{11} M_{\odot}$, the lowest halo mass scale for which we can probe halo occupation. The $z=0.36$ bin does exhibit unusual clustering and abundances that indicate sample variance is playing some role, but the redshift trends found in the both the quenched central and satellite galaxy populations is consistent with those found from SDSS results. Simply removing the $z=0.36$ results from consideration does not change any of the conclusions of this paper.

We thank the referee for many helpful comments and suggestions that have improved this work. This work was supported by World Premier International Research Center Initiative (WPI Initiative), MEXT, Japan. The HST COSMOS Treasury program was supported through NASA grant HST-GO-09822. We wish to thank Tony Roman, Denise Taylor, and David Soderblom for their assistance in planning and scheduling of the extensive COSMOS observations. We gratefully acknowledge the contributions of the entire COSMOS collaboration consisting of more than 70 scientists. More information on the COSMOS survey is available at http://cosmos.astro.caltech.edu/. It is a pleasure the acknowledge the excellent services provided by the NASA IPAC/IRSA staff (Anastasia Laity, Anastasia Alexov, Bruce Berriman and John Good) in providing online archive and server capabilities for the COSMOS data-sets.

\section{REFERENCES}

Abbas, U., de la Torre, S., Le Fèvre, O., et al. 2010, MNRAS, 406, 1306 Baldry, I. K., Glazebrook, K., \& Driver, S. P. 2008, MNRAS, 388, 945 Balogh, M. L., Navarro, J. F., \& Morris, S. L. 2000, ApJ, 540, 113 Behroozi, P. S., Conroy, C., \& Wechsler, R. H. 2010, ApJ, 717, 379 Behroozi, P. S., Wechsler, R. H., \& Conroy, C. 2013, ApJL, 762, L31 Bell, E. F., Wolf, C., Meisenheimer, K., et al. 2004, ApJ, 608, 752 Berlind, A. A., \& Weinberg, D. H. 2002, ApJ, 575, 587 Birnboim, Y., \& Dekel, A. 2003, MNRAS, 345, 349 Blanton, M. R., Hogg, D. W., Bahcall, N. A., et al. 2003, ApJ, 594, 186 
Bower, R. G., Benson, A. J., Malbon, R., et al. 2006, MNRAS, 370, 645 Brinchmann, J., \& Ellis, R. S. 2000, ApJL, 536, L77

Bundy, K., Ellis, R. S., Conselice, C. J., et al. 2006, ApJ, 651, 120

Bundy, K., Georgakakis, A., Nandra, K., et al. 2008, ApJ, 681, 931

Bundy, K., Scarlata, C., Carollo, C. M., et al. 2010, ApJ, 719, 1969

Cattaneo, A., Dekel, A., Devriendt, J., Guiderdoni, B., \& Blaizot, J. 2006, MNRAS, 370, 1651

Chabrier, G. 2003, PASP, 115, 763

Coil, A. L., Newman, J. A., Croton, D., et al. 2008, ApJ, 672, 153

Conroy, C., \& Gunn, J. E. 2010, ApJ, 712, 833

Conroy, C., Gunn, J. E., \& White, M. 2009, ApJ, 699, 486

Conroy, C., \& Wechsler, R. H. 2009, ApJ, 696, 620

Conroy, C., Wechsler, R. H., \& Kravtsov, A. V. 2006, ApJ, 647, 201

Conroy, C., Wechsler, R. H., \& Kravtsov, A. V. 2007, ApJ, 668, 826

Cool, R. J., Eisenstein, D. J., Fan, X., et al. 2008, ApJ, 682, 919

Cooper, M. C., Newman, J. A., Croton, D. J., et al. 2006, MNRAS, 370, 198

Cooray, A., \& Sheth, R. 2002, PhR, 372, 1

Croton, D. J., Springel, V., White, S. D. M., et al. 2006, MNRAS, 365, 11

Dekel, A., \& Birnboim, Y. 2006, MNRAS, 368, 2

Drory, N., Bundy, K., Leauthaud, A., et al. 2009, ApJ, 707, 1595

Geha, M., Blanton, M. R., Yan, R., \& Tinker, J. L. 2012, ApJ, 757, 85

Georgakakis, A., Coil, A. L., Willmer, C. N. A., et al. 2011, MNRAS, 418, 2590

George, M. R., Leauthaud, A., Bundy, K., et al. 2011, ApJ, 742, 125

George, M. R., Ma, C.-P., Bundy, K., et al. 2013, ApJ, 770, 113

Gunn, J. E., \& Gott, J. R. I. 1972, ApJ, 176, 1

Hansen, S. M., Sheldon, E. S., Wechsler, R. H., \& Koester, B. P. 2009, ApJ, 699, 1333

Hopkins, P. F., Cox, T. J., Kereš, D., \& Hernquist, L. 2008, ApJS, 175, 390

Hopkins, P. F., Cox, T. J., Younger, J. D., \& Hernquist, L. 2009, ApJ, 691, 1168

Hopkins, P. F., Croton, D., Bundy, K., et al. 2010, ApJ, 724, 915

Ilbert, O., Capak, P., Salvato, M., et al. 2009, ApJ, 690, 1236

Kauffmann, G., Heckman, T. M., White, S. D. M., et al. 2003, MNRAS, 341,54

Kereš, D., Katz, N., Fardal, M., Davé, R., \& Weinberg, D. H. 2009, MNRAS, 395,160

Kereš, D., Katz, N., Weinberg, D. H., \& Davé, R. 2005, MNRAS, 363, 2

Knobel, C., Lilly, S. J., Kovač, K., et al. 2013, ApJ, 769, 24

Kriek, M., van der Wel, A., van Dokkum, P. G., Franx, M., \& Illingworth, G. D. 2008, ApJ, 682, 896

Labbé, I., Huang, J., Franx, M., et al. 2005, ApJL, 624, L81

Leauthaud, A., Tinker, J., Behroozi, P. S., Busha, M. T., \& Wechsler, R. H. 2011, ApJ, 738, 45

Leauthaud, A., Tinker, J., Bundy, K., et al. 2012, ApJ, 744, 159

Li, C., \& White, S. D. M. 2009, MNRAS, 398, 2177

Madgwick, D. S., Somerville, R., Lahav, O., \& Ellis, R. 2003, MNRAS, 343,871

Maller, A. H. 2008, in ASP Conf. Ser. 396, Formation and Evolution of Galaxy Disks, ed. J. G. Funes \& E. M. Corsini (San Francisco, CA: ASP), 251

Mandelbaum, R., Seljak, U., Kauffmann, G., Hirata, C. M., \& Brinkmann, J. 2006, MNRAS, 368, 715

Marchesini, D., van Dokkum, P. G., Förster Schreiber, N. M., et al. 2009, ApJ, 701,1765

Moore, B., Lake, G., \& Katz, N. 1998, ApJ, 495, 139

More, S., van den Bosch, F. C., Cacciato, M., et al. 2011, MNRAS, 410, 210

Moster, B. P., Somerville, R. S., Maulbetsch, C., et al. 2010, ApJ, 710, 903

Moustakas, J., Coil, A. L., Aird, J., et al. 2013, ApJ, 767, 50

Muñoz-Cuartas, J. C., Macciò, A. V., Gottlöber, S., \& Dutton, A. A. 2011, MNRAS, 411, 584
Navarro, J. F., Frenk, C. S., \& White, S. D. M. 1997, ApJ, 490, 493

Noeske, K. G., Faber, S. M., Weiner, B. J., et al. 2007, ApJL, 660, L47

Peacock, J. A., \& Smith, R. E. 2000, MNRAS, 318, 1144

Peng, Y.-j., Lilly, S. J., Kovač, K., et al. 2010, ApJ, 721, 193

Peng, Y.-j., Lilly, S. J., Renzini, A., \& Carollo, M. 2012, ApJ, 757, 4

Phleps, S., Peacock, J. A., Meisenheimer, K., \& Wolf, C. 2006, A\&A, 457, 145

Pozzetti, L., Bolzonella, M., Zucca, E., et al. 2010, A\&A, 523, A13

Pozzetti, L., \& Mannucci, F. 2000, MNRAS, 317, L17

Purcell, C. W., Bullock, J. S., \& Zentner, A. R. 2007, ApJ, 666, 20

Quadri, R. F., Williams, R. J., Lee, K.-S., et al. 2008, ApJL, 685, L1

Robertson, B., Bullock, J. S., Cox, T. J., et al. 2006, ApJ, 645, 986

Sánchez, A. G., \& Cole, S. 2008, MNRAS, 385, 830

Scoccimarro, R., Sheth, R. K., Hui, L., \& Jain, B. 2001, ApJ, 546, 20

Scoville, N., Aussel, H., Brusa, M., et al. 2007, ApJS, 172, 1

Seljak, U. 2000, MNRAS, 318, 203

Skibba, R. A., \& Sheth, R. K. 2009, MNRAS, 392, 1080

Skibba, R. A., van den Bosch, F. C., Yang, X., et al. 2011, MNRAS, 410, 417

Stewart, K. R., Bullock, J. S., Barton, E. J., \& Wechsler, R. H. 2009, ApJ, 702, 1005

Strateva, I., Ivezić, Ž., Knapp, G. R., et al. 2001, AJ, 122, 1861

Tinker, J., Kravtsov, A. V., Klypin, A., et al. 2008a, ApJ, 688, 709

Tinker, J., Wetzel, A., \& Conroy, C. 2011, MNRAS, submitted (arXiv:1107.5046)

Tinker, J. L., Conroy, C., Norberg, P., et al. 2008b, ApJ, 686, 53

Tinker, J. L., George, M. R., Leauthaud, A., et al. 2012, ApJL, 755, L5

Tinker, J. L., Norberg, P., Weinberg, D. H., \& Warren, M. S. 2007, ApJ, 659,877

Tinker, J. L., Robertson, B. E., Kravtsov, A. V., et al. 2010a, ApJ, 724, 878

Tinker, J. L., Wechsler, R. H., \& Zheng, Z. 2010b, ApJ, 709, 67

Tinker, J. L., \& Wetzel, A. R. 2010, ApJ, 719, 88

van den Bosch, F. C., Yang, X., \& Mo, H. J. 2003, MNRAS, 340, 771

van den Bosch, F. C., Yang, X., Mo, H. J., et al. 2007, MNRAS, 376, 841

Velander, M., van Uitert, E., Hoekstra, H., et al. 2013, MNRAS, submitted (arXiv:1304.4265)

von der Linden, A., Wild, V., Kauffmann, G., White, S. D. M., \& Weinmann, S. 2010, MNRAS, 404, 1231

Wake, D. A., Nichol, R. C., Eisenstein, D. J., et al. 2006, MNRAS, 372, 537

Wake, D. A., Whitaker, K. E., Labbé, I., et al. 2011, ApJ, 728, 46

Wang, L., Li, C., Kauffmann, G., \& De Lucia, G. 2007, MNRAS, 377, 1419

Wechsler, R. H., Bullock, J. S., Primack, J. R., Kravtsov, A. V., \& Dekel, A. 2002, ApJ, 568, 52

Weinmann, S. M., van den Bosch, F. C., Yang, X., \& Mo, H. J. 2006, MNRAS, 366,2

Wetzel, A. R., Tinker, J. L., \& Conroy, C. 2012, MNRAS, 424, 232

Wetzel, A. R., Tinker, J. L., Conroy, C., \& van den Bosch, F. C. 2013a, MNRAS, 432, 336

Wetzel, A. R., Tinker, J. L., Conroy, C., \& van den Bosch, F. C. 2013b, arXiv:1303.7231

Williams, R. J., Quadri, R. F., Franx, M., van Dokkum, P., \& Labbé, I. 2009, ApJ, 691, 1879

Willmer, C. N. A., Faber, S. M., Koo, D. C., et al. 2006, ApJ, 647, 853

Yang, X., Mo, H. J., van den Bosch, F. C., Zhang, Y., \& Han, J. 2012, ApJ, 752, 41

Zehavi, I., Zheng, Z., Weinberg, D. H., et al. 2005, ApJ, 630, 1

Zehavi, I., Zheng, Z., Weinberg, D. H., et al. 2011, ApJ, 736, 59

Zheng, Z., Coil, A. L., \& Zehavi, I. 2007, ApJ, 667, 760

Zheng, Z., Zehavi, I., Eisenstein, D. J., Weinberg, D. H., \& Jing, Y. P. 2009, ApJ, 707,554

Zhu, G., Blanton, M. R., Burles, S. M., et al. 2011, ApJ, 726, 110 